

\title{
New Perspectives on Beltrami's Life and Work - Considerations Based on his Correspondence
}

\author{
Rossana Tazzioli
}

\section{To cite this version:}

Rossana Tazzioli. New Perspectives on Beltrami's Life and Work - Considerations Based on his Correspondence. Mathematicians in Bologna, 1861-1960, 2012, 10.1007/978-3-0348-0227-7_21 . hal01436965

\section{HAL Id: hal-01436965 \\ https://hal.science/hal-01436965}

Submitted on 22 Jan 2017

HAL is a multi-disciplinary open access archive for the deposit and dissemination of scientific research documents, whether they are published or not. The documents may come from teaching and research institutions in France or abroad, or from public or private research centers.
L'archive ouverte pluridisciplinaire HAL, est destinée au dépôt et à la diffusion de documents scientifiques de niveau recherche, publiés ou non, émanant des établissements d'enseignement et de recherche français ou étrangers, des laboratoires publics ou privés. 


\title{
New Perspectives on Beltrami's Life and Work - Considerations based on his Correspondence
}

\author{
Rossana Tazzioli \\ U.F.R. de Mathématiques, Laboratoire Paul Painlevé U.M.R. CNRS 8524 \\ Université de Sciences et Technologie Lille 1 \\ e-mail:rossana.tazzioliatuniv-lille1.fr
}

\begin{abstract}
Eugenio Beltrami was a prominent figure of 19th century Italian mathematics. He was also involved in the social, cultural and political events of his country. This paper aims at throwing fresh light on some aspects of Beltrami's life and work by using his personal correspondence. Unfortunately, Beltrami's Archive has never been found, and only letters by Beltrami - or in a few cases some drafts addressed to him - are available. In this paper some letters addressed by Beltrami to his foreign colleagues are published and annotated for the first time in order to give a more exhaustive picture of Beltrami's personality and to point out the impact of his work in Italy and abroad.
\end{abstract}

\section{Introduction}

Eugenio Beltrami (1835-1900) was one of the most influential mathematicians of the 19th century in Italy. One of the protagonists of the Risorgimento (the political movement preceding the Italian unification of 1861), he participated, together with other Italian mathematicians such as Enrico Betti (1823-1892), Francesco Brioschi (1824-1897), Felice Casorati (1835-1890), and Luigi Cremona (1830-1903), in the political and cultural life of post-unification Italy. Section 2 gives a picture of the involvement of Italian mathematicians in the Risorgimento and of their institutional commitments in the period immediately after unification.

Beltrami is well-known mainly for his works on differential geometry and non-Euclidean geometry, but he also made important contributions to mathematical physics over roughly thirty years of his scientific career. On his scientific work and the connection between geometry and mathematical physics several studies have already been published. ${ }^{1}$ This paper does not provide an exhaustive scientific biography of Beltrami; it aims rather at giving a fresh perspective on his life and work by considering his unpublished correspondence with some of his foreign colleagues.

As far as we know, no Beltrami Archive exist; however, many of his letters to Italian and foreign colleagues have already been published. ${ }^{2}$ In these letters new aspects of Beltrami's life and work are brought forth, such as his engagement with mathematics teaching and with institutional issues, his ideas on non-Euclidean geometry and its relations with Gaussian theory of surfaces, and his strong interest in mathematical physics.

In this paper we give an account of Beltrami's letters to Richard Dedekind (1831-1916), Pierre Duhem (1861-1916), Wilhelm Killing (1847-1923), Felix Klein (1849-1925), Rudolph Lipschitz (1832-

\footnotetext{
${ }^{1}$ For a scientific biography of Beltrami and especially for his work on non-Euclidean geometry see the Introduction to [10]; for his contribution to mathematical physics see [35] and [37].

${ }^{2}$ See for example Beltrami's letters to Hoüel in [10], to Betti, Tardy and Gherardi in [25], to Cesaro in [32], and to Chelini in [23].
} 
1903), and Gösta Mittag-Leffler (1846-1927), ${ }^{3}$ where it becomes very clear that Beltrami attributed great importance to mathematical physics both as a researcher and as a teacher charged with giving lectures on various areas of mathematical physics - on mechanics, elasticity, potential theory, the theory of electricity and magnetism (including Maxwell's equations), and the theory of heat. His interest in the new physics - as the Maxwell theory was called at the time $^{4}$ - is often confirmed in this correspondence, as well as his troubles due to the complexity and obscurity of this theory, which indeed had not yet been rigorously founded. These letters also show the strong influence of Beltrami's methods and results not only on geometry but also on mathematical physics. Furthermore, thanks to his high reputation in Italy and abroad, Beltrami's advice was generally followed by his colleagues. He often recommended skilled young mathematicians for positions or grants and he was often gratified to see his recommendation accepted.

Section 3 introduces Beltrami as a mathematician and as a political figure, and in particular points out what is new in the letters to his foreign colleagues published in the Appendix. In more detail, section 3.1 sketches Beltrami's life and scientific activity; section 3.2 aims at describing his opinion on Maxwell's electromagnetic theory, and how he managed to solve problems coming from this theory as a researcher and as a teacher; section 3.3 concerns his high reputation - some remarkable examples from his correspondence are shown, such as his engagement as a teacher, the influence of his research abroad, and his requests to participate Sonyain international undertakings. We attempt to express what Beltrami's letters highlight and how they contribute to a clarification of some aspects of his life and work.

Appendices 4.1-4.6 contain the unpublished correspondence between Beltrami and Dedekind (Appendix 4.1), and Beltrami's letters to Duhem, Killing, Klein, Lipschitz, and Mittag-Leffler (Appendices 4.2-4.5). Appendix 4.7 reproduces some letters exchanged between Cremona and Betti, and two letters from Beltrami to Betti concerning Sonya Kovalevskaya's affair of 1886, when several mathematicians tried to induce the Stockholm Academy of Sciences to accept Sonya Kovalevskaya (1850-1891) - though a woman - as a member of the Academy. Appendix 4.8 contains a Report written by Beltrami in 1889 and addressed to the University of Stockholm in support of Kovalevskaya. She was appointed full professor at the University of Stockholm the same year.

All the documents in the Appendices are annotated; the footnotes contain short biographies, bibliographical references, and some explanations when they are necessary.

\section{The Risorgimento Generation}

The history of modern Italy starts in 1861. In that year the various smaller states into which Italy had been politically and administratively divided were unified in a process called the Risorgimento. The government of Piedmont (the northwestern region of Italy, whose capital is Turin) obtained significant popular support which allowed it to become the leader of the idealistic and democratic mobilization against Austria. After popular uprisings, two wars of independence against Austria, and intense diplomatic activity, the Unification of Italy was finally achieved. Only in 1870, after the defeat of the papal army, did Rome become the capital of unified Italy.

Many Italian mathematicians - such as Beltrami, Betti, Brioschi, Casorati, Cremona, and others - participated as soldiers in the wars of independence, and as politicians in a general sense after unification. They raised the level of mathematical studies and, more generally, saw themselves as the builders of the new Italy. Betti was a volunteer and fought in Curtatone and Montanara in the battalion of the students of the University of Pisa; in 1848 Cremona participated in the defence of Venice against the Austrian regime, where he attained the ranks of corporal and then sergeant; Brioschi participated in the insurrection of Milan in 1848 against the Austrians and in 1870 he was involved in the taking of Rome. Beltrami and his family shared the ideals of the Risorgimento from the popular uprisings of 1848-49, as Beltrami wrote to Charles Hermite (1822-1901) in November 1887:

\footnotetext{
${ }^{3}$ These letters are unpublished except for the letter to Klein dated 17 April 1888 and the letter to Lipschitz dated 13 March 1877; both of them are published but not annotated in [10] on pages 211-213.

${ }^{4}$ Maxwell published his electromagnetic theory in the Treatise on Electricity and Magnetism in 1873 [30].
} 
[...] as a consequence of the political events of 48-49, the members of my family could no more expect to become civil servants of the Austrian government. [...] At the end of my studies - I had to renounce my doctorate for lack of means - [...] I had the good luck to become and remain for six years the private secretary of M. Didier, chief engineer of mines and the director of railways of the North Italy, an excellent man taking an almost fatherly care of me. In this period my vocation - a bit uncertain - turned to mathematical research. Since I was badly prepared by the feeble courses of that time, I began to learn the arithmetic of M. Serret.(My translation $)^{5}$

In 1853 Beltrami had indeed started to study mathematics at the University of Pavia, where he had attended Brioschi's lectures. After the working period described in this letter to Hermite, Beltrami continued his mathematical education in Milan where he met Cremona, who drove his interests to the geometry of curves and surfaces. Very soon Beltrami began to publish, and produced several interesting works on this subject. In 1862, thanks to Brioschi's intervention (Brioschi was the secretary of the Ministry of Public Education at that time), Beltrami became professor of algebra and analytic geometry at the University of Bologna, though he had never obtained his doctorate in mathematics.

In 1858 Betti, Brioschi and Casorati visited the universities of Göttingen, Berlin, and Paris, and came into contact with the most important mathematicians and institutes in Europe. They met Dedekind, Peter Gustav Lejeune Dirichlet (1805-1859), Bernhard Riemann (1826-1866), Leopold Kronecker (1823-1891), Karl Weierstrass (1815-1897), Joseph Bertrand (1822-1900), Hermite, and others. By tradition, Italian mathematics is supposed to have had its origins in this trip. The argument that the Risorgimento created a new starting point in mathematics - as well as in politics - obviously fits a patriotic ideology which emphasizes that the Unification of Italy had given wings to the aspirations and enthusiasm of the best minds in the country, in science no less than in other domains.

In 1908 in his inaugural address to the International Congress of mathematicians in Rome, Vito Volterra (1860-1940) claimed:

[...] it is then not surprising if, in the progress of science, a sudden change in Italian thought occurred, provoked by the quick development and spreading of this thought, and by the new qualities which it acquired and which made it richer in the years following the period of the political Risorgimento.(My translation) ([39], p. 59)

In reality, it is inconceivable that a mathematical school should have sprung up from nothing, or simply from a documentary trip. Even so, this view - extreme as it may appear - can still be taken as a suitable starting point, from which a deeper analysis may begin. ${ }^{6}$

Italian mathematicians were therefore in the front line of this process of nation-building, through the public and political offices they held. In particular, Beltrami and Casorati were senators; Brioschi and Betti were deputies, senators, and also under-secretaries in the Ministry of Education (in the period 1861-62 and 1874-76 respectively); Cremona was appointed Minister of Education in 1898, albeit only for one month, and was the director of the Scuola degli Ingegneri in Rome.

Brioschi was a key figure in the formation and guidance of the new entrepreneurial middle class of Northern Italy. This emerging class, which took shape in reaction against a sluggish and passive landowning class, expected much from the progress of science and technology. Therefore the mentality and outlook of many scientists were in perfect harmony with the ideas of this middle class and its desire to further and accelerate the growth of Italian industry. As an expression of this

\footnotetext{
5 “[... ] les événements politiques de 48-49 ayant mis ma famille au nombre de celles dont les membres ne pouvaient pas espérer un emploi public par le gouvernement autrichien, [... ] à la fin de mes études (en renonçant au doctorat, faute de moyens) [...] j'ai eu le bonheur de devenir et de rester, pendant six ans, secrétaire particulier de M. Diday, Ingénieur au chef des mines, Directeur de l'exploitation des chemins de fer de la Haute-Italie, excellent homme qui a eu pour moi des soins presque paternels. C'est dans cet intervalle que ma vocation un peu flottante, s'est décidée pour les études mathématiques et que, me trouvant décidément trop mal préparé par les faibles cours de ce temps là, j'ai entrepris de refaire toute mon instruction, à commencer par l'arithmétique de M. Serret." Letter from Beltrami to Hermite, Venise 1st November 1887; in Betti's Archive, Archivio della Biblioteca della Scuola Normale Superiore, Pisa.

${ }^{6}$ On the commitment of Italian mathematicians during the Risorgimento, see [33] and [12].
} 
shared project - matching the interests of the middle class and the orientation of mathematicians regarding their research and teaching - Brioschi founded the Polytechnic in Milan in 1863, with the intention of training engineers able to contribute to the development and modernization of Italy. ${ }^{7}$

The collaboration between Betti and Brioschi was the true driving force behind the initial development of Italian mathematics; it was extremely fruitful both because of its organizational impact and in consequence of the high-level research to which it led. The influence of Riemann - whom Betti met at the University of Göttingen for the first time - was crucial for Betti's scientific activity. ${ }^{8}$ Betti later invited Riemann to spend two years (1863-1865) at the University of Pisa, when Beltrami was professor of geodesy at the same university. Betti deeply involved himself in promoting Riemann's ideas not only in Italy but also in Europe at large.

Generally speaking, Italian mathematicians tried to build Italian national identity by following several directions:

(i)Developing new research fields;

(ii) Creating new mathematical chairs and new research institutes - such as the Polytechnic of Milan;

(iii) Starting the publication of new mathematical high-level journals able to compete with German or French mathematical journals - such as the Annali di Matematica pura ed applicata in 1858 and the Giornale di Matematiche in $1863 ;^{9}$

(iv) Increasing the number of grants for young mathematicians who aimed at specializing in foreign universities, and especially in Germany and France;

(v) Reforming university and secondary school;

(vi) Writing or translating good mathematical treatises for mathematical teaching.

Just after the celebrated trip throughout Europe, Betti translated Riemann's Inauguraldissertation on the theory of complex functions [34] and spread interest in this field of research in Italy, to which especially Casorati made important contributions. Beltrami published his important works on non-Euclidean geometry ([3], [4]) employing the methods of differential geometry he had learned when he was a student in Pavia. Cremona's geometrical research led to the emergence of the so-called Italian school of algebraic geometry.

They also engaged themselves in writing new treatises on different subjects - the theory of determinants (Brioschi, [15]), real and complex analysis (Ulisse Dini (1845-1918) [22] and Casorati [16]), rational mechanics (Domenico Chelini (1802-1878) [18]), graphical statics (Cremona [20]), potential theory (Betti [9]), and many others. Beltrami did not publish any treatise but, as it appears from reading his letters (see section 3.2), he often drafted his courses on mathematical physics. One of these course contains his lectures on the theory of elasticity held at the University of Rome; it was handwritten by one of his student, Alfonso Sella (1865-1907), and is held by the Library of the Department of Mathematics in Genoa. Other lectures on mathematical physics held by Beltrami at the University of Pavia in the period 1881-1883 are contained in the Dipartimento di Matematica in Pavia. These lectures concern heat theory, magnetism and electromagnetism, potential theory, electrodynamics, and elasticity of solid bodies.

Betti was the director for about thirty years of the celebrated Scuola Normale Superiore of Pisa, where many mathematicians studied and were trained in mathematical research . Among them we may mention Dini, Salvatore Pincherle (1853-1936), Luigi Bianchi (1859-1928), Gregorio Ricci Curbastro (1853-1925), and Volterra. Pincherle spent a year (1877-1878) at the University of Berlin with Weierstrass; Bianchi and Ricci Curbastro attended Klein's lectures at the University of Munich in 1879-1880 and in 1877-1878 respectively.

The Risorgimento generation had the great merit of creating the conditions for the development of the second generation of mathematicians, who made Italy a great power in mathematics, ranking only after France and Germany. This period can be called the golden age of Italian mathematics; the mathematicians of the next generation would have to measure up to this high standard.

\footnotetext{
${ }^{7}$ On Brioschi and the Polytechnic of Milan, see [29].

${ }^{8}$ For Betti and his mathematical school at the University of Pisa, see [11].

${ }^{9}$ On these mathematical journals see [13] and [17].
} 


\section{New Perspectives}

\subsection{A Biographical Sketch}

Beltrami studied with Brioschi at the University of Pavia from 1853 to 1856, when he had to leave Pavia before graduating because of his Risorgimento ideals. ${ }^{10}$ Just after the unification of Italy he was offered the chair of complementary algebra and analytic geometry at the University of Bologna, which he held during the academic year 1862-63. In 1863 Ottaviano Mossotti (1791-1863), professor of geodesy at the University of Pisa, died; therefore Betti asked Beltrami to come to Pisa to take over Mossotti's chair and Beltrami accepted. From 1866 to 1873 he was back in Bologna, where he taught rational mechanics. In 1873 he accepted an offer to join his old colleague and friend Cremona in Rome, where Cremona was the director of the Scuola degli Ingegneri and Beltrami held the chair of rational mechanics at the university. In 1876, Beltrami moved to Pavia where he taught mathematical physics. In 1891 he returned to Rome, where he taught until his death. In 1898 he became the president of the Accademia dei Lincei and, the following year, a senator of the Italian Kingdom.

Beltrami's correspondence allows us to highlight academic motivations and personal reasons which led him to change his university and his teaching direction several times. ${ }^{11}$

Beltrami is mainly known for his contribution to differential geometry, which continued the works of Gauss and Riemann, and to non-Euclidean geometry. These two research fields were indeed deeply connected, as Beltrami himself often claimed. In his Saggio di interpretazione della geometria non euclidea [3], Beltrami shows that hyperbolic geometry could be represented on a particular surface of the Euclidean space having constant negative curvature (called the pseudospherical surface). By using a suitable lexicon, where "geodesic line on the pseudospherical surface" stands for straight line of the hyperbolic plane" and "angle between two geodesic lines" for "angle between two straight lines", Beltrami translates all the theorems that are valid in the Lobachevskij geometry to theorems valid on the pseudospherical surface. However, his model of the hyperbolic plane in Euclidean space is valid only locally.

His second fundamental paper on non-Euclidean geometry, Teoria fondamentale degli spazi di curvatura costante [4], originates from a deep analysis of Riemann's work on differential geometry and rigorously proves some important results concerning Riemannian geometry. [3] and [4] were soon translated into French and gave an impulse to the development of non-Euclidean geometry in all Europe.

In the 1870s, Beltrami changed his research field and directed his interests to mathematical physics - first to potential theory and then, from 1882 onward, to the theory of elasticity. However, he also published many works concerning rational mechanics, electricity and magnetism. The mathematical tools employed in his research on mathematical physics are those of differential geometry. Beltrami, like other mathematicians and physicists of the 19th century, tried to explain propagation of physical phenomena in space by imagining an ether filling the whole universe - to a particular strain system of the ether corresponds a phenomenon which is transmitted through space by contact (and not at-a-distance) from one ether particle to an immediately contiguous one.

It was also a (natural-)philosophical motivation which led Beltrami to prove new and interesting results, such as the Laplace-Beltrami equation in potential theory (which generalizes the classical Laplace equation to an $n$-dimensional Riemannian manifold); the equations of elastic equilibrium in a space with constant curvature; and the necessary and sufficient conditions for six given functions to constitute the components of an actual elastic deformation - these formulae led Beltrami to deduce the equations of elastic equilibrium expressed by elastic stress, which are nowadays called "Beltrami-Mitchell's equations". 12

Beltrami and Betti are the two key figures of mathematical physics in Italy in the second half of the 19th century. Both were members of university and ministerial commissions, engaged in politics,

\footnotetext{
${ }^{10}$ The director of the Collegio Ghislieri in Pavia expelled Beltrami for political reasons; see for example [28].

${ }^{11}$ In particular, see Beltrami's letters to Hoüel in [10] and his (unpublished) letters to Betti contained in Betti's Archive, Archivio storico della Scuola Normale Superiore di Pisa; Beltrami's letters to Betti will be published in [25]. On Beltrami's life and work, see also [37].

${ }^{12}$ On this subject see [35].
} 
education, and mathematical teaching as well as in mathematical research. As already mentioned Betti was the director of the Scuola Normale Superiore of Pisa and trained many mathematicians in research - among them I shall mention Luigi Bianchi (1859-1928), Gregorio Ricci Curbastro (18531925), and Vito Volterra (1860-1940). On the contrary, Beltrami did not found an actual school of geometry and/or of mathematical physics - his frequent changes of town and university probably prevented him from creating a stable group of students and researchers working in the same university and on similar subjects. Nevertheless, his research on geometry and on mathematical physics had a great influence in Italy and abroad, as these letters show. In Italy Valentino Cerruti (1850-1909), Ernesto Cesaro (1859-1906), Gian Antonio Maggi (1856-1937), Ernesto Padova (1845-1896), and Carlo Somigliana (1860-1955) followed Beltrami's ideas and researches, even though sometimes they were not his students in the usual sense. His letters to his foreign colleagues reproduced in the Appendices show how his works - not only on non-Euclidean geometry, but also on mathematical physics - were well-known and appreciated also abroad.

\subsection{The New Physics}

As we have already pointed out, since the 1870 s Beltrami turned his interests from geometry to mathematical physics, and published about fifty papers on potential theory, the theory of elasticity, hydrodynamics, electrostatics, electrodynamics, magnetism, and electromagnetism. In his papers Beltrami often combined mathematical physics and non-Euclidean geometry, and obtained remarkable results. In particular, he successfully applied his geometrical research to the generalization of some aspects of mechanics, potential theory and theory of elasticity to non-Euclidean spaces. Beltrami's ideas are well described in the following passage by Maurice Lévy:

M. Beltrami is indeed a follower of Faraday and Maxwell; that is to say, he aims at suppressing any assumption of action at-a-distance and at explaining physical phenomena by means of a medium connecting all natural bodies, so that one can not touch a body without producing a more or less strong effect on the others.(My translation) ${ }^{13}$

In Beltrami's opinion, in fact, an ethereal medium existed, which was able to propagate forces in space by means of its own deformations. Strains and tensions of the ether were submitted to the laws of the theory of elasticity. As already mentioned, Beltrami published various papers on Maxwell's equations and tried to give a mechanical interpretation of electromagnetic phenomena. For example, in a paper published in 1882 [5], he described a system of elastic deformations of ether "in accordance with" the ideas of Faraday and Maxwell. Explaining electric and magnetic phenomena by means of an ethereal and elastic fluid allowed the reduction of electromagnetism to mechanics - and that was considered as an important achievement by mathematicians and physicists of the 19th century.

Between 1884 and 1886, Beltrami published three papers ([6], [7], [8]) on Maxwell's electromagnetic theory and its mechanical interpretation. On January 4th, 1885 Beltrami explained to Ludwig Schläfli (1814-1895) that he was very interested in Maxwell's electromagnetic theory and also his university lectures dealt with this subject. In particular, he tried to connect together electric and elastic phenomena. He wrote to Schläfli:

This year I am especially interested in the theory of elasticity, both in my lectures and in my research. This theory is very interesting to me, also from the point of view of the method. In particular, I am investigating a question connected to Maxwell's ideas, which tends to establish a deep connection between electric and elastic phenomena. Until now my deductions seem to be rather contrary to the possibility of this connection, but I do not dare to formulate conclusive statements - Maxwell himself did not want to attribute

\footnotetext{
13 "Mr Beltrami, en effet, est un disciple de Faraday et de Maxwell, c'est-dire que sa tendance est d'arriver à la suppression de l'hypothèse des actions à distance et à l'explication des phénomènes physiques par la présence d'un milieu servant de lien entre tous les corps de la nature, de telle sorte qu'on ne puisse pas toucher à l'un d'eux, sans que tous les autres s'en ressentent plus ou moins." This unpublished report of Maurice Lévy concerns Beltrami's election as a corresponding member of the Paris Academy of Sciences, and is contained at the Archives de l'Académie des Sciences in Paris.
} 
a precise form to his interpretation and hoped for a long time, unfortunately in vain, to get more concrete results. (My translation) ${ }^{14}$

Beltrami referred to his attempt to justify Maxwell's electromagnetic theory by deducing the ether deformations able to produce Maxwell's equations. In a paper published in 1886 [8] Beltrami found a negative answer: he proved that no elastic medium could produce strains and tensions in accordance with Maxwell's electromagnetic theory. Nevertheless, he believed in the validity of Maxwell's theory and was one the first Italian scientists who actively studied the new physics.

In a letter to Duhem dated July 25th, 1891, Beltrami confessed he feared that he misunderstood the ideas of the illustrious "English physicist". In another letter to his French colleague (15 April 1892) Beltrami wrote how his aim was to justify Maxwell's results, though "maybe there does not exist in the history of science another example of a doctrine so badly digested and explained as the theory contained in the famous Treatise". (My translation) ${ }^{15}$

In various papers Beltrami extended potential theory, the theory of elasticity, and classical mechanics to non-Euclidean spaces. He indeed considered the possibility that physical phenomena could propagate in a non-Euclidean space. This idea was shared by the German mathematicians Killing, Lipschitz, and Ernst Schering (1824-1889), who tried to extend certain classical theories of mathematical physics - such as Hamiltonian mechanics and potential theory - to a Riemannian manifold with constant curvature. ${ }^{16}$ The long letter by Beltrami to Killing (March 22nd, 1888) shows that his German colleague essentially shared Beltrami's research program. Beltrami suggested him to study physical laws concerning mutual interactions between electric particles in non-Euclidean spaces that could replace Ampère's law in the usual space. Beltrami thought that this research, and more in general research concerning electro-dynamic theory in non-Euclidean spaces, could lead to "very interesting results".

However, in Italy mathematical physics was not very popular at that time, as Beltrami complained in some of his letters. On July 25th, 1891 Beltrami wrote to Duhem that he felt himself "very isolated" in his research on mathematical physics. And in a letter to Klein (April 17th, 1888) he deplored the attitude of Italian physicists towards mathematical physics. According to Beltrami, "only two or three physicists" knew the fundamental theories of mathematical physics in Italy. And he added:

As to the fear you seem to feel concerning an increasing divorce between mathematics and physics, I would dare to say that Germany is, in my opinion, the country where this divorce seems to me the less advanced. (My translation $)^{17}$

In the same letter, Beltrami remarked that it could be profitable if a journal of mathematical physics were to exist. In fact, such a journal could help those who are usually considered as mathematicians among physicists and as physicists among mathematicians. It is evident that Beltrami referred to his own experience.

\subsection{Beltrami's Reputation}

Beltrami had strong international relationships and often met foreign colleagues, as it is shown by his letters. For example, he met Schläfli several times between 1871 and 1873; in 1873 he met Hermann

\footnotetext{
14 "In questo anno mi occupo specialmente, tanto nelle lezioni, quanto nel mio studio privato, della teoria dell'elasticità, la quale mi interessa molto, anche dal lato del metodo. In particolare sto studiando una questione che si collega con quelle vedute di Maxwell le quali tenderebbero a stabilire un nesso intimo fra i fenomeni elettrici e gli elastici. Finora le mie deduzioni sarebbero piuttosto contrarie alla possibilità di questo nesso, ma non ardirei ancora di formulare conclusioni assolute, tanto più che lo stesso Maxwell non ha voluto dare una forma precisa alla sua interpretazione ed ha sperato, disgraziatamente invano, di ottenere da tempo risultati più concreti." Letter by Beltrami to Schläfli, in [26], p. 120-121.

${ }^{15}$ The original French is: “il n'y a peut-être pas d'exemple, dans l'histoire de la science, d'une doctrine aussi mal digérée et exposée, que celle du célèbre Treatise".

${ }^{16}$ On these works see [36].

17 "Quant à la crainte que Vous semblez concevoir d'un divorce toujours croissant entre les mathématiques et la physique, j'oserais vous dire que l'Allemagne est, à mon avis, le pays où ce divorce me paraîtrait le moins avancé". Letter by Beltrami to Klein, in [10], p. 213.
} 
von Helmholtz (1821-1894) for the first time and again in 1877; during his summer holidays he met Kronecker, Georg Ferdinand Frobenius (1849-1917), Thomas Archer Hirst (1830-1892), Carl Friedrich Geiser (1843-1934), Jacob Rosanes (1842-1922) and others. In a letter to Betti (30 August 1879) Beltrami was happy to write that he has joined "Schläfli, Geiser, Frobenius, Meyer, Hirst, Cremona, Casorati" and that at that moment "they were waiting for Zeuthen". In another letter to Betti (17 September 1880) he wrote that he had to keep Hermann Amandus Schwarz (1843-1921) company. ${ }^{18}$

In spite of the isolation he felt, Beltrami was a reference figure in Italy as well as abroad. I shall show some examples. Dedekind asked Beltrami for information about an Italian mathematician Aureliano Faifofer (1843-1909) - who intended to translate Dirichlet's treatise on number theory into Italian. This work had been edited by Dedekind himself and published in 1863. Beltrami's answer convinced Dedekind to accept Faifofer's proposal and the Italian translation of Dirichlet's classical book appeared in 1881 (see the correspondence between Dedekind and Beltrami in Appendix 4.1).

Beltrami was asked to participate in prestigious celebrations: he was invited by Schwarz to take part in the centenary celebration of Gauss's birth held in Göttingen in 1877. In the end Beltrami had to forego participation in the celebrations. ${ }^{19}$ Beltrami was also asked to participate in the scientific meeting celebrating the 70th birthday of Charles Hermite (see Beltrami's letter to MittagLeffler dated June 27th, 1892). Mittag-Leffler often invited Beltrami to publish his papers in Acta Mathematica, the well-known Swedish journal which Mittag-Leffler had founded and directed for many years.

As we have already mentioned, his frequent changes of university and teaching area prevented Beltrami from creating his own mathematical school. Nevertheless his letters show his commitment to raise the level of Italian mathematics. He always supported the best students, and considered mathematical teaching an important issue. In a letter to Betti (20 February 1882) he praised Enrico d'Ovidio (1843-1933) "because he is able to train good students for research; something which did not occur in Turin for a long time". (My translation) ${ }^{20}$ In another letter to Betti (13 March 1881) he recommended Gian Antonio Maggi, one of his students at the University of Pavia, who possessed all the requisites necessary for "becoming a good physicist".

His high reputation allowed Beltrami to write to his colleague Felix Klein in order to introduce to him some young Italian mathematicians who aimed at doing further specialized work in Germany. In a letter dated 7 December 1883 Beltrami introduced to Klein Giacinto Morera (1856-1909) and in another letter (5 January 1885) he wrote to him: "I take this opportunity to thank you for your kindness towards M. Morera and the other young Italian people who have preceded him in Leipzig" ("Je saisis cette occasion pour vous remercier aussi de la bienveillance que vous avez bien voulu témoigner à M.r Morera, ainsi qu'aux autres jeunes gens italiens qui l'ont précédé à Leipzig.")

Beltrami addressed Klein again in order to introduce another young Italian researcher, the physicist Alfonso Sella, who was the son of Quintino Sella. The latter was a mineral engineer and an "eminent man", a well-known politician who also became Ministry of Finance.

Furthermore, Mittag-Leffler asked for Beltrami's intervention on a delicate question concerning the Russian mathematician Sonya Kovalevskaya. She had difficulty finding an academic position, though she was en excellent mathematician - in 1874 she obtained her PhD with a dissertation supervised by Weierstrass, and in 1888 she won the Prix Bordin proposed by the Academy of Sciences of Paris. In 1884 she obtained a position of extraordinary professor at the University of

\footnotetext{
${ }^{18}$ See Beltrami's letters to Schläfli dated 6 August 1871, 17 August 1871, and 20 September 1873, in [26], p. 113-116; Beltrami's letter to Mittag-Leffler dated 19 July 1883; Beltrami's letter to Schwarz dated 5 April 1877. Beltrami's letters to Schwarz are in the Archiv der Berlin-Brandeburgischen Akademie der Wissenschaften of Berlin, Nachlass Schwarz.

${ }^{19}$ That can indeed be read in Beltrami's letter to Schwarz dated April 25th, 1877: "Le circostanze alle quali alludevo nella mia lettera precedente mi hanno definitivamente obbligato a privarmi del piacere di assistere alla solennità scientifica del 30 aprile. Nel mentre mi reco a dovere di ciò significarLe, per ringraziarLa dei cortesi eccitamenti venutami da Lei, faccio appello alla di Lei benevolenza per pregarLa di attestare ai geometri tedeschi la viva partecipazione che io prendo, benché lontano, ai sentimenti di ammirazione e di gratitudine che li fanno convenire a Gottinga per onorare la memoria del grand'uomo che, sebbene avesse applicato a sé stesso il motto Nil actum reputans, si quid superesset agendum, ha pur tuttavia lasciato tanto di actum da rendere ardua ai posteri la via dell'agendum." This letter is unpublished (see footnote 18).

${ }^{20}$ The original Italian is: "In quanto al D'Ovidio debbo dire [...] che egli merita lode anche perché gli riesce di fare buoni allievi, addestrandoli alla ricerca; cosa che a Torino da molto tempo non si vedeva."
} 
Stockholm for five years. Mittag-Leffler asked Beltrami to support the cause of Kovalevskaya in order to obtain for her a permanent position. Answering Mittag-Leffler's request, Beltrami wrote the Report some days later. ${ }^{21}$ Finally, Kovalevskaya obtained a professorship at the University of Stockholm in 1889. Unfortunately she died two years later, in 1891. The letter addressed by Beltrami to the University of Stockholm, where he referred on Kovalevskaya's scientific works, is reproduced in Appendix 4.8 .

We point out that another international undertaking had already taken place in 1886, when Weierstrass proposed Kovalevskaya for a vacancy at the Academy of Sciences of Stockholm. However only men were accepted as members of the Stockholm Academy. Though many mathematicians supported Sonya Kovalevskaya, the project was finally abandoned. ${ }^{22}$

Appendix 4.7 contains two letters from Beltrami to Betti, written in 1886: as Beltrami explained, Angelo Genocchi (1817-1889) and Betti asked him and Casorati to sign a document supporting the cause of Kovalevskaya. But he hesitated and, apparently, Casorati shared his opinion. Indeed, as Beltrami himself confessed, he did not know what this document contained exactly and he had never read Kovalevskaya's works carefully. Furthermore, Beltrami thought that German mathematicians, who better knew Kovalevskaya and her mathematical skill, had to support her more strongly. Finally, Beltrami and Casorati preferred to avoid any personal involvement in this matter.

We may point out that other Italian mathematicians, such as Betti and Cremona, supported Kovalevskaya's cause since 1886. A letter from Betti to Cremona (26 March 1886) and Cremona's answer are reproduced in the same Appendix 4.7. Betti informed Cremona about an international undertaking in favour of Kovalevskaya, and said that many French mathematicians - such as Hermite, Camille Jordan (1838-1922), Gaston Darboux (1842-1917), Paul Appel (1855-1930), Henri Poincaré (1854-1912), Emile Picard (1856-1941), and Félix Tisserand (1845-1896) - joined it. In his answer (2 April 1886) Cremona wrote that he intended to support Kovalevskaya, together with Betti, Genocchi and other Italian mathematicians.

\section{Appendix}

\subsection{The correspondence Beltrami-Dedekind}

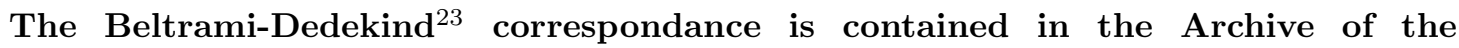
Niedersächsische Staats-und Universitätsbibliothek, Göttingen, Cod. Ms. Dedekind XIII: 2

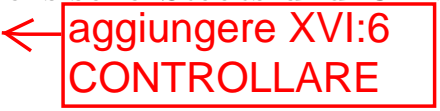

Letter n. 1

Draft by Richard Dedekind to Eugenio Beltrami

An Herrn Ritter Eugenio Beltrami, Professor an der K. Universität in Rom

Hochgeehrter Herr Professor! Hiermit erlaube ich mir, Ihnen eine Bitte um gefällige Auskunft vorzutragen, deren Veranlassung die folgende ist. Gestern habe ich ein Schreiben des Herrn Faifofer ${ }^{24}$ Professor am Lyceum zu Venedig, welcher mir seine Absicht zu erhennen gibt, eine italienische Übersetzung der "Vorlesungen über Zahlentheorie von Lejeune Dirichlet" herauszugeben, und mir

\footnotetext{
${ }^{21}$ See Beltrami's letter to Mittag-Leffler dated 16 May 1889.

${ }^{22}$ See [19], p. 109, [1], p. 162-163.

${ }^{23}$ Richard Dedekind (1831-1916) studied at the universities of Göttingen and Berlin. At the University of Göttingen he followed Dirichlet's lectures on number theory, which he collected and published in 1863 . In 1858 he began teaching at the Polytechnic of Zürich and in 1862 came back to his native Braunschweig, where he taught at the Technische Hochschule. He made important contributions to abstract algebra, algebraic number theory and the foundations of the real numbers.

${ }^{24}$ Aureliano Faifofer (1843-1909) studied at the University of Padua, where he spent some years as assistant professor. In 1868 he was appointed professor at the Liceo Foscarini in Venice. He published several mathematical textbooks, translated into many languages.
} 
zugleich mittheilt, ${ }^{25}$ dass Sie, hochgeehrter Herr Professor, und ebenso Herr Professor Bellavitis ${ }^{26}$ zu Padua seinen Plan gebilligt haben. Die Ausführung derselben wird mir, dem deutschen Herausgeber, sehr angenehm sein, aber nur in der Voraussetzung, dass die Übersetzung sich mit vollem Verständniss und möglichst treu dem Originale anschliesst. Da mir nun der Name des Herrn Professor Faifofer bisher noch nicht bekannt gewesen ist, so werden Sie es natürlich finden, wenn ich noch vor der erforderlichen Verhandlung mit dem hiesigen Verleger Vieveg bitte mir vertraulich mitzutheilen, ob Sie Herrn Faifofer näher kennen und für vollkommen fähig halten, ein solches, keineswegs leichtes Unternehmen gut auszuführen; es würde mich auch besonders interessieren zu erfahren, ob Herr Faifofer, der mir über seine eigene Person gar nichts schreibt, sich bisher schon mit der Theorie der Zahlen ernstlich beschäftigt hat, oder ob er erst jetzt ein eigentliches Studium derselben beginnen will. Dies Letztere würde zwar in meinen Augen kein absolutes Hinderniss sein, wenn Sie ihn im Übrigen als einen guten Mathematiker kennen, aber es würde mich veranlassen die Bedingung zu stellen, dass ich die Übersetzung vor dem Druck genau durchsehen und eventuell auch Abänderungen derselben verlangen dürfte.

Die Ausführung des Plans, welchen ich mit Freude begrüsse, würde für mich zugleich eine willkommene Veranlassung sein, die italienische Sprache gründlicher als bisher zu erlernen. Freilich bin ich wohl im Stande, die ausgezeichneten Arbeiten der jetzigen italienischen Mathematiker zu verstehen, und ich erlaube mir bei dieses Gelegenheit, Ihnen, hochgeehrter Herr Professor, meinen herzlichen Dank für die höchst interessanten und werthvollen Geschenke auszusprechen, durch welche Sie mich hoch erfreut haben; allein weiter gehen meine Kenntnisse bisher nicht, und aus diesem Grunde habe ich mich in meiner Muttersprache an Sie gewendet, deren Sie, wie aus Ihren Arbeiten hervorgeht, vollständig mächtig sind. Sie werden mich zu grossem Danke verpflichten, wenn Sie meine Bitte erfüllen und mich mit einer italienischen Antwort beehren wollen.

Mit ausgezeichneter Hochachtung verbleibe ich Ihr ganz ergebener

R. Dedekind, Professor

Braunschweig,

7 Juni 1876

Petrithorpromenade 24

\section{Letter n. 2 \\ Eugenio Beltrami to Richard Dedekind}

Roma 13 Giugno 1876

Chiarissimo Signor Professore

Mi affretto a soddisfare al desiderio manifestato nella graditissima di Lei lettera del 7 corr., jeri soltanto pervenuta nelle mie mani.

Io conosco il Sig.r D.r Faifofer già da qualche anno, senza però avere (anche per la lontananza delle nostre rispettive sedi) una grande intimità con lui, vedendolo io di quando in quando soltanto nelle ferie autunnali.

Rispetto però allo scopo delle informazioni da Lei desiderate, credo di poterLe fornire alcuni elementi abbastanza sicuri, che dividerò in due parti, cioè in quella che si riferisce alla cognizione della lingua tedesca, ed in quella che si riferisce alla competenza matematica.

In quanto alla prima parte, credo di potere assicurare che il prof. Faifofer conosce bene la lingua anzidetta, e la parla correntemente, avendo anche più volte viaggiato in Germania. D'altronde la

\footnotetext{
${ }^{25}$ Johann Peter Gustav Lejeune Dirichlet (1805-1859) was professor at the University of Berlin from 1831 to 1855 , when after Gauss's death he became professor at the University of Göttingen. His lectures on number theory were edited and published by Dedekind, who was one of his students at the University of Göttingen: Dirichlet, P.G., Vorlesungen ber Zahlentheorie, Braunschweig, Vieweg, 1863. The Italian translation of this book was actually published by Aureliano Faifofer, Lezioni sulla teoria dei numeri di P. G. Lejeune Dirichlet, pubblicate e corredate di appendice da R. Dedekind, translated and edited by A. Faifofer, Venezia, Tipografia Emiliana, 1881.

${ }^{26}$ Giusto Bellavitis (1803-1880) was professor at the University of Padua from 1845 onwards, though he did not attend any establishment of higher education. Faifofer was one of his students. Bellavitis is well-known for his works on equipollencies, a kind of vector calculus.
} 
precisa e ristretta fraseologia matematica non presenta alcuna delle difficoltà che lo stile letterario può presentare talvolta anche a chi possiede profondamente una lingua straniera.

In quanto alla conoscenza dell'argomento delle ormai celebri Vorlesungen del Dirichlet, di cui Ella ha così largamente accresciuto il valore pedagogico e scientifico colla propria illuminata collaborazione, mi riesce più difficile pronunziare un giudizio esplicito. Il Sig.r Faifofer è conosciuto in Italia come autore di due recenti trattati elementari, l'uno di Aritmetica, l'altro di Algebra, i quali, senz'avere alcuna pretesa di originalità, anzi essendo forse troppo rigidamente calcati sui programmi e sulle istruzioni Ministeriali (relative all'insegnamento matematico nei Ginnasi e Licei), sono però da collocarsi fra i migliori di quelli fin qui pubblicati per uso delle scuole, ed hanno già trovato buona accoglienza. ${ }^{27}$ Inoltre, il medesimo Sig.r Faifofer è considerato, e giustamente, come uno dei migliori insegnanti di matematica elementare, tanto dal punto di vista della chiarezza quanto da quello della esattezza e del rigore delle sue lezioni.

Ma altre prove del suo sapere in matematica, e specialmente nelle parti più elevate della scienza, non esistono (almeno a mia conoscenza); né, da quanto egli stesso mi comunicò intorno agli studii universitarii da lui fatti, mi risulta ch'egli abbia in essi approfondita particolarmente qualche disciplina matematica superiore. Mi ricordo perfettamente d'avere udito, dalla sua bocca, che stava da ultimo studiando le Vorlesungen, e che, per suo esercizio, le traduceva. Più tardi poi mi parlò delle difficoltà che aveva dovuto superare nell'andare pi innanzi, e dell'intenzione che cominciava a formare di dare pubblicità alla sua traduzione, intenzione alla quale io non ho certamente mancato di far plauso. Sono ora circa due anni che ebbe luogo la prima di queste comunicazioni; e, sebbene io non creda che il Faifofer abbia allargato i suoi studii sulla teoria dei numeri (ricorrendo ad altri autori più o meno speciali), inclino tuttavia a ritenere, specialmente dopo il passo fatto presso di Lei, che egli abbia esattamente compreso il classico libro da lui tradotto, e che, riservandosi Ella la revisione dei fogli (come non posso a meno di consigliarLe), la versione italiana sia per riescire soddisfacente.

Io Le sono grato a ogni modo delle di Lei gentili righe, che mi hanno fornito l'occasione di dirigerLe questa mia, e mi professo con tutta stima e devozione

obbl.mo E. Beltrami

\section{Letter n. 3 Eugenio Beltrami to Richard Dedekind}

Pavia 9 Marzo 1879

Illustrissimo Signore

La ringrazio distintamente del gentilissimo dono ch'Ella si è compiaciuto di farmi della III ed. delle Vorlesungen ueber Zahlentheorie, divenute ormai classiche per il felice innesto delle di Lei proprie elucubrazioni sul tronco originale delle Lezioni di Dirichlet. Io ammiro queste profonde e delicate ricerche, quantunque non abbia finora avuto il coraggio di affrontare i problemi.

Con questa di Lei pubblicazione, e con quelle dei Sig.ri Meyer e Grube, ${ }^{28}$ credo sia presso a poco esaurito il campo così largamente fecondato dalle lezioni orali di Dirichlet. Credo però anche che, per coronare l'opera, bisognerebbe fare per lui quello che è stato fatto per Riemann ${ }^{29}$ e per altri eminenti matematici, cioè ripubblicarne in un sol corpo le Memorie e gli scritti sparsi. Ho ferma

\footnotetext{
${ }^{27}$ In 1876, when Beltrami wrote the letter, Faifofer had already published the following books for secondary schools: Elementi di geometria, Venezia, 1878; Elementi d'algebra e trigonometria : ad uso dei licei, Venezia, 1878.

${ }^{28} \mathrm{He}$ refers to Dirichlet's lectures edited by Franz Grube (1835-1893) with the title Vorlesungen über die im umgekehrten Verhältnisse des Quadrats der Entfernung wirkenden Kräfte, Leipzig, Teubner, 1876. Another edition of Dirichlet's lectures has been published by G. Arendt, Vorlesungen über die Lehre von den einfachen und mehrfachen Bestimmten Integralen, Braunschweig, Vieweg, 1904. Oscar Emil Meyer (1834-1909) edited Franz Neumann's lectures on the theory of elasticity: Vorlesungen über die Theorie der Elasticität der festen Körper und des Lichtäthers gehalten in der Universität Königsberg, Leipzig, B. G. Teubner, 1885.

${ }^{29} \mathrm{He}$ refers to Riemann's collected works edited by H. Weber and published in 1876 for the first time (Leipzig, Teubner). Bernhard Riemann (1826-1866) - a personal friend of Dedekind - was professor at the University of Göttingen from 1859 until his death.
} 
opinione che una tal pubblicazione sarebbe estremamente gradita a tutto il mondo matematico. La perfezione di forma che distingue i lavori di Dirichlet li rende eminentemente adatti ad essere, dirò così, popolarizzati fra gli studiosi, e resi accessibili anche a chi non ha opportunità di fruire delle pubbliche Biblioteche.

RinnovandoLe i miei più sinceri ringraziamenti, La prego di aggradire l'espressione della mia profonda stima e di credermi sempre di Lei div.mo obbl.mo

Eugenio Beltrami

\subsection{Letters by Eugenio Beltrami to Pierre Duhem}

The letters by Eugenio Beltrami to Pierre Duhem, ${ }^{30}$ Dossier Duhem, B-10, Archives de l'Académie des Sciences, Paris

\section{Letter n. 1}

\section{Eugenio Beltrami to Pierre Duhem}

[Undated letter]

Eugenio Beltrami Professore nella R. Università di Roma offre ses remerciements les plus sincères à M. le prof. Duhem pour son précieux cadeau.

\section{Letter n. 2 \\ Eugenio Beltrami to Pierre Duhem}

[Undated letter]

Eugenio Beltrami exprime à M. Duhem ses meilleurs souhaits pour la nouvelle anné, le félicitant de son infatigable activité scientifique et faisant ses meilleurs voeux pour l'heureux succès de son oeuvre de savant et de professeur.

E. B.

Letter n. 3

\section{Eugenio Beltrami to Pierre Duhem}

Pavia 25 Juillet 1891

Monsieur et très honor Collègue

Je viens de recevoir, aujourd'hui même, par l'entremise de M. Gauthier-Villars, le beau volume, formant la 1.ère partie d'un Traité d'électricité et de magnétisme, que Vous avez bien voulu m'adresser. ${ }^{31}$ Je tiens à Vous remercier de la manière la plus distinguée de cet offre si bienveillant et si considérable, et qui m'est d'autant plus précieux que son sujet est celui sur lequel se concentre mon attention en ce moment. D'après ce que Vous dîtes dans Votre préface, touchant les méthodes

\footnotetext{
${ }^{30}$ Pierre Duhem (1861-1916) studied at the Ecole Normale Supérieure in Paris. He was professor at the universities of Lille (1887-1893), Rennes (1893-1894) and Bordeaux (1894-1916). He strongly contributed to mathematics, and to history and philosophy of science. His scientific works concern thermodynamics, hydrodynamics, elasticity and chemical physics. His works on history of science mainly concern history of mechanics and, more generally, history of mathematical physics.

${ }^{31}$ Duhem, P., Leçons sur l'électricité et le magnétisme, 3. vols., Paris, Gauthier-Villars, $1891-1892$.
} 
et les points de vue qui Vous sont propres, j'ai la certitude que j'apprendrai beaucoup de choses par Votre livre, surtout du côté thermodynamique, que, je Vous l'avoue, je n'ai jamais eu l'occasion d'approfondir, autant que l'importance su sujet le mérite. Je me trouve malheureusement trèsisolé depuis longtemps, par rapport aux études physico-mathématiques, et l'occasion me manque d'éclaircir mes idées sur plusieurs points délicats, que je suis obligé, par là, à laisser de côté.

Etant sur le point de partir pour la campagne, j'ai fait à la hàte un paquet de quelques unes de mes publications antérieures et je vais Vous l'adresser, en Vous priant de vouloir bien les agréer comme une faible marque de mon estime et de ma reconnaissance. Il y en aurait bien d'autres que je voudrais Vous faire parvenir, mais je ne les ai pas sous ma main en ce moment: j'en tiendrai note pour mon retour, si tant est que ce retour ait lieu: car c'est presque décidé que j'irai à Rome, pour la nouvelle année scolaire, et dans ce cas j'aurai sur le bras la grosse affaire d'un déménagement complet!

Vous ne parlez pas des théories de Maxwell, et en effet Vous n'aviez pas besoin d'en parler, au point de vue des sujets que Vous traitez dans ce premier volume. C'est dans le second que Vous la rencontrerez, et je désire beaucoup voir la place que Vous lui ferez. Je dis cela, car mon éternelle préoccupation est de bien comprendre, si c'est possible, la pensée de l'ingénieux physicien anglais.

Veuillez agréer, Monsieur et honoré collègue, l'expression de mes sentiments de considération la plus distinguée.

Votre bien obligé E. Beltrami

P. S. Voulez-Vous bien me permettre une rectification méticuleuse? Dans la 2.e Note à la page 3 de Votre Traité c'est la 1.ère série du Journal de Physique qu'il faut indiquer.

\section{Letter n. 4}

\section{Eugenio Beltrami to Pierre Duhem}

Rome 27 Décembre 1891

\section{Monsieur et très honoré Collègue}

Les vicissitudes de mon changements de résidence m'ayant amené à force retenir à Pavie tous les envois d'imprimés, qui m'ont été adressés dans ces derniers mois, ce n'est qu'aujourd'hui même que j'ai reçu les nombreuses publications, en partie nouvelles, que Vous avez eu la bonté de m'envoyer à différentes reprises. Je regrette ce retard à double titre: d'abord parce que je n'ai pu Vous présenter plus tôt mes remerciements les plus sincères, ce dont je Vous demande bien pardon; ensuite parce que j'aurais pu tirer grand avantage de quelques unes de ces publications, pour une étude sur l'électromagnétisme, que je viens de rédiger pour l'Académie de Bologne. ${ }^{32}$ Mais je tâcherai de suppléer à cet inconvénient dans l'intervalle assez long qui s'écoulera avant l'impression.

Je n'ai presque vu que les titres et les sujets principaux de Vos ouvrages, et j'aurai l'occasion de Vous en parler plus au long dans la suite, car plusieurs de ces sujets m'intéressent au plus haut degré. Permettez-moi cependant de Vous offrir, dès à présent, mes compliments les plus sincères pour cette imposante masse de travail accumulée en aussi peu d'années, dans plusieurs directions différentes, dont quelques unes ont été frayées la première fois par Vous.

Parmi Vos gracieux envois il y a plusieurs doubles. Ce sont les Mémoires suivants:

Sur l'équivalence des courants et des aimants;

Sur les dissolutions d'un sel magnétique;

Sur les équations générales de la thermodynamique;

Des corps diamagnétiques;

Sur la continuité entre l'état liquide et l'état gazeux et sur la théorie générale des vapeurs. ${ }^{33}$

\footnotetext{
${ }^{32}$ Beltrami, E., Considerazioni sulla teoria matematica dell'elettromagnetismo, Memorie della R. Accademia delle Scienze dell'Istituto di Bologna, s. 5), 2, 1892, p. 313-378; in [2] 4, p. 436-498.

${ }^{33}$ Duhem, P., Sur l'équivalence des courants et aimants, Annales scientifiques de l'Ecole Normale Supérieure, s, 3 6, 1899, p. 297; Sur les dissolutions d'un sel magnétique, Ibid., s. 3, 7, 1899, p. 289; Sur les équations générales de la thermodynamique, Ibid., s. 3, 8, 1891, p. 231; Des corps diamagnétiques, Travaux et Mémoires des Facultés de Lille 1, n. 2, 1889; Sur la continuité entre l'état liquide et l'état gazeux et sur la théorie générale des vapeurs, Ibid. 1, n. $5,1891$.
} 
Si ce n'est que par mégarde que ces Mémoires m'ont été adressés en double exemplaire, je Vous les renverrai tout-de-suite: Vous n'avez qu'à me le dire. Si c'est avec intention, j'en ferai l'usage que Vous voudrez bien m'indiquer. J'ai même pensé que Vous m'aviez peut-être écrit à ce sujet: mais je n'ai reçu aucune lettre de Vous. Malheureusement les nombreuses tournées que j'ai dû faire récemment, légitiment le doute que quelques lettres se soit perdu.

Je n'ai pas encore de logement fixe proprement dit: je suis encore à la recherche. En attendant mon adresse est: Institut Royal de Physique, Panisperna 89.

Veuillez bien agréer, excellent collègue, mes sentiments les plus distingués et les plus affectueux.

E. Beltrami

\section{Letter n. 5 \\ Eugenio Beltrami to Pierre Duhem}

Rome 15 Avril 1892

Monsieur et Collègue très-honoré!

Je ne suis que trop en retard avec les remerciements que je Vous dois, et que je Vous offre de la manière la plus distinguée, pour l'envoi du troisième volume de votre Traité, ${ }^{34}$ ainsi que pour celui de Votre Discours. Veuillez pardonner ce retard, qui n'est dû qu'à une suite malheureuse de préoccupations douloureuses que j'ai eu, depuis le commencement de cette année, pour la santé toujours chancelante de ma compagne et qui m'ont d'autant plus affecté que c'était surtout à cause de cela que je m'étais déterminé à accepter la nouvelle résidence de Rome, jugée plus favorable à la santé de M.me Beltrami.

La manière tout-à-fait nouvelle dont Vous posez, dans Votre nouveau volume, la question fondamentale des actions électrodynamiques mérite un examen sérieux, que je n'ai pu jusqu'ici qu'initier. J'ai pu, au contraire, puiser des connaissances précieuses dans divers chapitres détachés de ce volume, ayant rapport à certains sujets particuliers dont j'avais besoin de m'occuper. J'espère pouvoir me familiariser un peu plus tard avec les considérations d'ordre plus général qui caractérisent Votre ouvrage: mais je voudrais auparavant en finir avec la révision des épreuves (qui [...] on ne peut plus en longueur) d'un Mémoire sur l'électromagnétisme ${ }^{35}$ où, comme Vous verrez, j'ai laissé dominer des tendances en quelques sorte opposées, soit en ne considérant que des systèmes continus de courants, soit en renonçant le plus possible à l'invocation des lois dites élémentaires. Je dois ajouter cependant que mon cadre était bien plus restreint que le Vôtre, et que mon but principal était de justifier plusieurs résultats de Maxwell, dont moi-même j'avais longtemps été sans ne rendre bien compte. Les confirmations que j'ai trouvées me disposent, je l'avoue, à croire que l'on pourra également justifier d'autres parties d'une théorie que Vous me semblez juger moins favorablement. Mais je puis Vous assurer que je suis tout prêt à me raviser: et je Vous accorde, en tous cas, qu'il n'y a peut-être pas d'exemple, dans l'histoire de la science, d'une doctrine aussi mal digérée et exposée, que celle du célèbre Treatise. ${ }^{36}$

En attendant je vais Vous adresser quelques petites brochures, dont l'une se rapporte de nouveau au principe de Huygens. ${ }^{37}$ J'ai notablement simplifié la démonstration que Vous savez, de manière, surtout, à rendre inutile toute discussion touchant l'existence des intégrales: il me semble que le nouveau procédé se prête encore mieux aux exigences didactiques. J'y ai rattaché quelques développements ultérieurs, dont Vous saisirez facilement l'esprit.

Veuillez agréer, Monsieur et excellent collègue, l'expression renouvelée de ma reconnaissance, ainsi que celle de mon estime la plus sincère et la plus affectueuse.

E. Beltrami

\footnotetext{
${ }^{34}$ See footnote 31.

${ }^{35}$ See footnote 32 .

${ }^{36}$ Maxwell, J.C., A Treatise on Electricity and Magnetism, 2 vols., Oxford, Clarendon, 1873.

${ }^{37}$ Beltrami, E., Sull'espressione analitica del principio di Huygens, Rendiconti della R. Accademia dei Lincei, 1 , 1892, p. 99-108; in [2] 4, p. 499-509.
} 
Letter n. 6

Eugenio Beltrami to Pierre Duhem

Rome 6 Xbre 1898

Monsieur et Collègue très-honoré!

J'éprouvais depuis longtemps le plus vif désir de Vous offrir mes remerciements les plus sincères et les plus empressés pour le gracieux envoi de Votre 3. me volume de la Mécanique chimique, ${ }^{38}$ et ce sentiment s'est encore augmenté à la suite de l'arrivée d'un nouveau paquet de Vos travaux les plus récents, dont la seule inspection des sujets suffit pour faire apprécier de plus en plus la bonté de Vos choix, d'un côté, et l'extraordinaire puissance de travail, de l'autre, puissance qui se révèle aussi dans l'oeuvre dont Vos assistants puisent la conception et les méthodes dans l'étude et l'imitation de la Vôtre.

Malheureusement depuis quelque temps ma santé, qui s'était bien rétablie, ou paraissait l'être, s'est troublée de nouveau, par la répétition, quoique atténuée, des accidents qui l'avaient fortement compromises il y a deux ans, et j'ai dû me mettre tout-à-fait au repos, en renonçant non seulement à la reprise de mes leçons, mais à toute espèce de travail intellectuel, et ce n'est que depuis quelques jours que les médecins me permettent ma correspondance, que je dois borner, cependant, d'après leur terminologie, à sa partie non-scientifique. Je commence cependant à espérer, si tout continue de marcher comme à présent, de pouvoir reprendre en Janvier au moins une partie de mes occupations.

Quoiqu'il en soit [... ] pourquoi il ne m'a pas été donné de Vous faire parvenir plus tôt l'expression de mes sentiments de reconnaissance, ainsi que de Vous prier de vouloir bien présenter mes remerciements bien distingués à Votre excellent disciple M. Marchis. ${ }^{39}$ Je serai bien heureux si je pourrai encore recouvrer la faculté de suivre autrement que par le désir et les souhaits les développements et les progrès que Vous apportez à nos connaissances de la mécanique des phénomènes.

Veuillez agréer les salutations cordiales de Votre bien obligé

E. Beltrami

\section{Letter n. 7}

\section{Eugenio Beltrami to Pierre Duhem}

Rome 6 Juillet 1899

Monsieur et Collègue très-honoré

Je regrette infiniment d'avoir dû tant retarder à Vous exprimer mes remerciements, et plus encore mon admiration sincère et profonde, pour le gracieux envoi du dernier volume de Votre Ouvrage sur la mécanique chimique. ${ }^{40}$ Je suis malheureusement trop peu versé dans la chimie moderne pour être à même d'apprécier le détail de Vos recherches sur les différents sujets que Vous avez traités, mais je puis assez suivre la ligne générale de Vos raisonnements et admirer la souplesse que Vous avez su donner aux procédés d'analyse et de déduction rationnelle, pour acquérir la conviction que Vous avez rendu un grand service à la science et fait faire de beaux progrès à sa coordination et à son enseignement!

Comme je Vous je le disais tout à l'heure, je regrette bien vivement de ne pas avoir eu moyen de prendre plus tôt connaissance de Votre important volume, mais d'abord ma santé n'est plus la même qu'au temps passé et tout travail un peu suivi me fatigue beaucoup, et ensuite mes fonctions académiques, dans les deux derniers mois, m'ont donné bien des tracas, et m'en donnent encore. En outre (et cela Vous surprendra beaucoup) depuis quelques semaines le Roi a voulu me nommer Sénateur, et quoique mes connaissances ne me donnent pas beaucoup d'avantages pour l'exercice de cette nouvelle fonction, il n'en est pas moins vrai qu'elle me prend assez de temps et qu'elle détourne matériellement (pour ainsi dire) mon attention de ce qui a trait aux études et à la science

\footnotetext{
${ }^{38}$ Duhem, P., Traité élémentaire de mécanique chimique, fonde sur la thermodynamique, 4 vols., Paris, Hermann, 1897-1899.

${ }^{39}$ Lucien Marchis (1863-1941), professor of Aviation at the Faculty of Sciences of Paris.

${ }^{40}$ See footnote 38 .
} 
pure. Quoique il en soit je suis bien heureux d'arriver encore à temps pour Vous féliciter de Votre oeuvre savante et géniale et Vous souhaiter les meilleurs succès dans le monde scientifique.

Votre bien obligé et bien dévoué

E. Beltrami

\subsection{A letter by Beltrami to Wilhelm Killing}

This letter by Eugenio Beltrami to Wilhelm Killing ${ }^{41}$ is contained in Nachlass Killing, N. 8, Universitäts-und Landesbibliothek, Münster

Pavia 22 Marzo 1888

Chiarissmo collega

Una disgrazia che ho avuto nella famiglia, ${ }^{42} \mathrm{mi}$ ha impedito di rispondere prima d'ora alla gradita di Lei lettera del 2 di questo mese, e di darLe, in ispecie, l'assicurazione che non solo io, ma tutti gli studiosi delle scienze matematiche, hanno per Lei tutta quella considerazione e quella simpatia che vengono giustamente tributate a chi lavora con costanza e con successo per il progresso della scienza.

Rilevo dalla di Lei lettera che Ella avrebbe intenzione, dopo il termine delle difficili ricerche in cui è attualmente impegnato e di cui ho ricevuto con gratitudine i saggi già pubblicati, di studiare la teoria dell'attrazione degli ellissoidi nello spazio non euclideo. Questo sarà certamente un interessante soggetto di studio: tuttavia, conoscendosi già la forma che assume nel detto spazio la legge newtoniana, ${ }^{43}$ è probabile che l'interesse del lavoro si riporti soltanto sulla natura analitica delle nuove formole che Ella incontrerà nel risolvere i problemi già trattati nello spazio euclideo.

Senza quindi volerLa distogliere da questa intrapresa, io mi permetterei di indicargliene un'altra, la quale potrebbe forse condurre a conseguenze più inaspettate, e sarebbe la ricerca della legge che, nello spazio non euclideo, fa le veci di quella d'Ampère, relativamente all'azione mutua delle correnti elettriche, ed in generale lo studio della teoria elettrodinamica nel detto spazio. ${ }^{44}$ Non sarei alieno dal credere che in questa via si potessero incontrare dei risultati molto interessanti. L'unico tentativo che è stato già fatto (a mia conoscenza) in questo genere di argomenti è quello del Sig.r Prof.re Ernesto Schering, ${ }^{45}$ il quale si è occupato di ricerche molto generali intorno alla legge di Weber. ${ }^{46}$ Ma, come Ella sa, la validità di questa ultima legge è molto controversa, mentre la legge d'Ampère e quelle trovate dal venerando F. Neumann sono universalmente riconosciute come inattaccabili, od almeno come basi necessarie di qualunque teoria: cosicché sarebbero queste che si dovrebbero,

\footnotetext{
${ }^{41}$ Wilhelm Killing (1847-1923) studied at the universities of Münster and Berlin. In 1892 he was appointed professor at the University of Münster. His first research concerns the theory of surfaces and non-Euclidean geometry; later on he contributed to the theory of transformation groups and to Lie algebras.

${ }^{42}$ Most probably Beltrami refers to the death of his aunt living in Padoa.

${ }^{43}$ Some of Beltrami's papers concern potential theory in non-Euclidean spaces; in particular, he explicitly deduced the potential function in a space with constant negative curvature in [6]; on the subject see [37].

${ }^{44}$ Ampère's law, discovered by André-Marie Ampère in 1826, relates the magnetic field around a closed loop to the electric current passing through the loop.

${ }^{45}$ Possibly Beltrami referred to the paper by Ernst Christian Julius Schering (1824-1889), professor at the University of Göttingen, "Zur mathematischer Theorie elektrisch. Ströme" published in the fourth volume (1857) of Annalen der Physik und Chemie.

${ }^{46}$ Starting from Ampère's law, Wilhelm Eduard Weber (1804-1891) obtained a general fundamental law of electrical action, expressing the force between moving charges. His fundamental electrical law depends upon the relative velocities and the relative accelerations of the particles. The Coulomb electrostatic law thus becomes a special case of Webers general law, when the particles are at relative rest. Weber's contributions to electrodynamics appeared in the Elektrodynamische Maasbestimmungen (Berlin, Springer, 1893) which collect seven long works published from 1846 to 1878 . See [21].
} 
innanzi tutto, riportare allo spazio non euclideo. ${ }^{47}$ Potrebbe darsi, del resto, che Ella conoscesse qualche lavoro, a me ignoto, già pubblicato sull'argomento, nel qual caso ritirerei la mia proposta e la convertirei nella preghiera di darmi, con Suo comodo, qualche notizia su tali lavori già compiuti da altri.

Gradisca i sensi della mia affettuosa stima e mi creda sempre

di Lei dev.mo

Eugenio Beltrami

\subsection{Letters by Eugenio Beltrami to Felix Klein}

The letters by Eugenio Beltrami to Felix Klein ${ }^{48}$, Cod. Ms. Klein 8, 79, Niedersächsische Staats-und Universitätsbibliothek, Göttingen). Letter 3 is published, but not annotated, in ([10], p. 213).

\section{Letter n. 1}

\section{Eugenio Beltrami to Felix Klein}

Pavie 7 Xbre 83

Monsieur et vénéré collègue

Je prends la liberté de Vous présenter M.r le Docteur G. Morera, ${ }^{49}$ qui vient à Leipzig pour y faire des études mathématiques. Je vous serai infiniment obligé si vous voudrez bien lui faire un accueil bienveillant et lui donner les secours et les conseils dont il pourra avoir besoin. Je suis sûr qu'il fera tout son possible pour mériter vos soins. Il s'est occupé surtout, avec succès, des recherches sur les équations de la dynamique: aussi j'espère que vous pouvez le recommander à M.r le prof.r Mayer, ${ }^{50}$ que je n'ai pas l'honneur de connaître personnellement.

M.r Gerbaldi ${ }^{51} \mathrm{~m}$ 'a dit que Vous avez été incommodé dans la santé, il y a quelques mois. Je veux espérer que vous vous portiez tout-à-fait bien maintenant et je vous souhaite de tout mon coeur un rétablissement complet, si votre état de santé vous laisse encore quelque chose à désirer.

Je Vous prie de vouloir bien présenter mes hommages à M.me votre femme, à laquelle ma femme aussi fait ses compliments, et j'ai l'honneur de me dire

votre bien obligé E. Beltrami

\footnotetext{
${ }^{47}$ Franz Ernst Neumann (1798-1895), the father of Carl Neumann, studied at the universities of Berlin and Jena. In 1825 he became Privatdozent and in 1829 professor at the University of Königsberg. Neumann established mathematically the laws of induction of electric currents in some papers published in the 1840s. As a starting point he took Lenz's proposition (1834) according to which the current induced in a conductor moving in the vicinity of a current or a magnet will flow in the direction that tends to oppose the motion. Considering Ampère's law for a closed circuit, Neumann was led to what it is known as the mutual potential of two circuits - the amount of mechanical work that must be performed against the electromagnetic forces in order to carry the two circuits to an infinite distance, when the currents are maintained unchanged. See [21].

${ }^{48}$ Felix Klein (1849-1925) studied at Göttingen, Berlin, and Paris. He was appointed professor at the University of Erlangen in 1872; later on he moved to the Technische Hochschule of Munich (1875-1880), and then to the universities of Leipzig (1880-1886) and Göttingen (1886-1913). His main research concern geometry, theory of algebraic equations and function theory. He also published important treatises on elementary mathematics and history of mathematics.

${ }^{49}$ Giacinto Morera (1856-1909) studied at Turin, Pisa, Pavia, Leipzig and Berlin. Beltrami, Klein and Weierstrass were among his teachers. In 1886 he became professor of rational mechanics at the University of Genoa, and in 1901 succeeded Vito Volterra at the University of Turin. His main research concerns complex analysis, but he also gave contributions to analytical mechanics, thermodynamics, and elasticity.

${ }^{50}$ Christian Gustav Adolph Mayer (1839-1908) studied at the Universities of Leipzig, Göttingen, Heidelberg and Königsberg. He taught mathematics at the University of Heidelberg all his life. He studied the theory of differential equations, the calculus of variations and mechanics. From 1871 to 1907 he exchanged many letters with Klein.

${ }^{51}$ Francesco Gerbaldi (1858-1934) studied at the University of Turin, and specialized at the University of Pavia and in Germany. In 1890 he was appointed professor of geometry at the University of Palermo, where he collaborated with Giuseppe Bagnera, Michele De Franchis, and Michele Cipolla on questions of geometry and algebraic geometry.
} 
Letter n. 2

Eugenio Beltrami to Felix Klein

Pavie, 5 Janvier 85

Monsieur et cher collègue

Je vous prie de vouloir bien m'excuser si je ne Vous ai pas encore offert mes remerciements pour l'obligeant envoi des vos Vorlesungen ueber das Ikosaeder. ${ }^{52}$ Mais j'ai été pendant plusieurs mois préoccupé à cause de la santé de ma femme, et ce n'est que depuis peu que je suis heureusement délivré de toute inquiétude. Votre livre a sans doute fait beaucoup de plaisir à ceux qui sont au courant des théories élévées auxquelles il se rapporte: mais il en fait un plus grand encore à tous ceux qui, comme moi, ont eu la malheur de rester en dehors de ce grand courant, car il va leur permettre de s'y orienter, à l'aide d'un guide dont on ne pouvait pas trouver le meilleur. Aussi peu de livres auront ils un plus sympathique accueil.

Je saisis cette occasion pour vous remercier aussi de la bienveillance que vous avez bien voulu témoigner à M.r Morera, ainsi qu'aux autres jeunes gens italiens qui l'ont précédé à Leipzig.

Je vous prie de vouloir bien présenter mes hommages à M.me Klein, de la part de ma femme aussi, et d'agréer l'expression de mes sentiments de reconnaissance et d'admiration.

votre bien dévoué E. Beltrami

\section{Letter n. 3 \\ Eugenio Beltrami to Felix Klein}

Pavie 17 Avril 1888

Très cher et honoré collègue ${ }^{53}$

J'ai reçu avec grand plaisir votre chère lettre du 15, et je me suis aussitôt occupé de prendre des mesures afin que le programme que Vous m'avez envoyé soit inséré dans le Cimento et dans les Comptes Rendus des Lincei: j'espère que cela se fera au plus tôt. ${ }^{54}$ Cependant si Vous étiez à même de m'envoyer quelques autres exemplaires du programme, je m'en servirais peut-être utilement pour le faire connaître à des professeurs qui pourraient à leur tour exciter quelques uns de leurs meilleurs élèves à s'occuper du sujet.

J'ai été bien content d'apprendre que Vous vous intéressez à présent aux questions de physique mathématique: elles ne pourront que gagner en passant par vos mains et je m'attends à quelques uns de vos heureux tours de force.

Quant à la crainte que Vous semblez concevoir d'un divorce toujours croissant entre les mathématiques et la physique, j'oserais vous dire que l'Allemagne est, à mon avis, le pays où ce divorce me paraîtrait le moins avancé: peut-être certaines parties de la science n'y sont pas encore dévenues assez populaires, pour ainsi dire, parmi les physiciens, mais en revanche il y en a plusieurs autres qui sont entrées assez bien dans le domaine commun. Que diriez Vous donc si vous voyiez de près ce qui se passe en Italie, où deux ou trois physiciens seulement ont une connaissance approfondie des théories fondamentales!

Une chose qui m'a toujours surpris c'est qu'au milieu de tant de publications périodiques, dont plusieurs ont un caractère très-spécial, personne n'ait jamais songé à fonder un Recueil pour la physique mathématique: ce qui pourrait être très-avantageux surtout pour les physiciens, qui ont souvent quelque répugnance à consulter les journaux mathématiques, et dont quelques uns n'en ont pas même le moyen. Seulement il faudrait qu'un pareil Recueil eusse une allure un peu moins rigide que de coutume: qu'on y fit de la place à des bonnes Monographies sur les sujets les moins généralement connus, à des Recensions dûment développées, à des discussions scientifiques, destinées surtout à détruire les préventions, quelques fois même assez fondées, contre l'emploi de l'analyse en

\footnotetext{
${ }^{52}$ Klein, F., Vorlesungen über das Ikosaeder und die Auflösung der Gleichungen vom 5. Grade, Leipzig, 1884.

${ }^{53} \mathrm{Je}$ vous écris en français dans la pensée de vous causer moins de fatigue. (Footnote by Beltrami)

${ }^{54}$ Most probably Beltrami refers to Klein's Erlangen Program which was translated into Italian by Gino Fano, Considerazioni comparative intorno a ricerche geometriche recenti, Annali di Matematica, s. 2, 17, 1889, p. 307-343.
} 
général, ou d'une certaine analyse en particulier. Un tel Recueil contribuerait peut-être à rendre moins désagréable la position de beaucoup de ceux qui s'adonnent à de semblables études, et dont le sort est assez souvent celui de passer pour physicien parmi les mathématiciens, et pour mathématicien parmi les physiciens, et de n'être écouté, en définitive, ni par les uns ni par les autres.

La nouvelle que Vous me donnez de la présence chez Vous d'un des fils du regretté Sella m'a beaucoup intéressé: ${ }^{55}$ j'aurai probablement connu ce jeune homme lorsqu'il était encore enfant, car j'allais souvent, à Rome, chez son père, un homme éminent dont je garde un souvenir des plus chers et dont la perte a été un véritable malheur pour notre pays. Je fais des voeux sincères pour que ce fils fasse honneur à sa naissance.

Veuillez agréer, mon excellent ami, mes salutations les plus affectueuses, et présenter mes hommages à M.me Klein: veuillez aussi offrir mes compliments à vos honorables collègues Riecke et Voigt. 56

votre bien dévoué E. Beltrami

\subsection{Letters by Beltrami to Rudolph Lipschitz}

The letters by Eugenio Beltrami to Rudolph Lipschitz ${ }^{57}$ are contained in Nachlass Lipschitz, Mathematisches Institut, Bonn. Letter 3 is published, but not annotated, in [10], p. 211-212.

\section{Letter n. 1}

\section{Eugenio Beltrami to Rudolph Lipschitz}

Bologna 18 Novembre 1872

\section{Chiar.mo Sig. Professore}

Mi prendo la libertà di inviarLe una mia fotografia, nella speranza ch'Ella voglia accoglierLa con quella gentilezza con cui si compiace di accettare i miei piccoli lavori, e di favorirmi le tanto importanti sue produzioni. Se poi Le fosse possibile, ora o più tardi, di farmi un dono della sua propria fotografia, io me ne terrei onorato come di favore veramente singolare. Voglia continuarmi la di Lei benevolenza ed aggradire i sentimenti della mia più profonda considerazione.

Dev.mo aff.mo Eugenio Beltrami

Ormai tutti piangono la morte immatura del grande Clebsch. ${ }^{58}$ Possa, questo universale compianto, confortare la sua povera famiglia!

\footnotetext{
${ }^{55} \mathrm{He}$ refers to Alfonso Sella (1865-1907) who studied elasticity, radioactivity, acoustics and crystallography. His father was the Italian politician Quintino Sella.

${ }^{56}$ Eduard Riecke (1845-1915) studied mathematics and physics at the universities of Stuttgart, Tübingen and Göttingen. In 1873 he was appointed professor of physics in Göttingen. He was interested in different fields of physics and mathematical physics, such as magnetism, hydrodynamics, thermodynamics, electricity, and crystallography. He also published an important textbook of physics (Lehrbuch der Physik, 1896). Woldemar Voigt (1850-1919) studied at the universities of Leipzig and Königsberg, where he became extraordinary professor of physics in 1875 . In 1883 he was appointed full professor at the University of Göttingen, with the promise that he and Riecke were to have a new physical institute (which was not ready until 1905). Voigt studied crystallography and its connection to the theory of elasticity. In 1908 he introduced the word tensor in the vocabulary of mathematical physics in relation to his research on crystals.

${ }^{57}$ Rudolph Lipschitz (1832-1903) studied at the universities of Königsberg and Berlin. He taught at secondary schools in Königsberg and from 1857 at the University of Berlin. In 1862 he was appointed professor at the University of Breslau and in 1864 moved to the University of Bonn. His publications concern different field of mathematics and mathematical physics: analysis, mechanics, number theory, theory of differential equations, potential theory and elasticity. His well-known treatise on the foundations of analysis, Grundlagen der Analysis (Bonn, 1877-1880), is mentioned in the letters.

${ }^{58}$ Rudolf Friedrich Alfred Clebsch (1833-1872) studied in Königsberg and Berlin. His scientific career started at the University of Berlin and ended at the University of Göttingen. Together with Carl Neumann he founded the journal Mathematische Annalen. His main works concern the theory of differential equations, invariant theory, and algebraic geometry.
} 
Letter n. 2

Eugenio Beltrami to Rudolph Lipschitz

Bologna 12 Luglio 1873

Egregio signor professore

A suo tempo ebbi la graditissima sua lettera del 25 Maggio, e, posteriormente, l'Estratto francese delle di Lei memorie sulle funzioni differenziali, di cui sommamente La ringrazio. ${ }^{59}$

Io posso assicurarLa che l'onore derivante all'Accademia dalla nomina di Lei a socio corrispondente non è stato sentito ed apprezzato soltanto da me; ${ }^{60}$ ma da tutti quelli che si interessano alle scienze esatte nell'Accademia stessa, e l'unanimità colla quale fu accolta la relativa proposta, mostra che questo sentimento era penetrato anche negli altri soci. Io deploro che gli Statuti attualmente in vigore, e non ancora informati ai bisogni del nostro tempo, limitino i diritti dei Corrispondenti al ricevimento del solo volumetto dei rendiconti annuali. Ma ho fondata speranza che alla prima revisione dei detti Statuti (che risalgono ancora all'epoca del Governo pontificio!) si introdurrà, fra molte altre, anche la regola di mandare ai Corrispondenti esteri la collezione delle Memorie in 4to.

Ho già fatto introdurre, nella nota dei Soci, la correzione relativa al di Lei prenome. Credo che un consimile errore sia stato commesso, e parimenti corretto, riguardo al di Lei collega sig. prof. Clausius. ${ }^{61}$

La prego di aggradire i miei cordiali e rispettosi saluti, e di volermi ricordare al sig. Maestro Wasiliewsky. ${ }^{62}$

Di Lei Dev.mo

aff.mo E. Beltrami

\section{Letter n. 3}

\section{Eugenio Beltrami to Rudolph Lipschitz}

Illustre collega

Ho ricevuto con vera gratitudine il cospicuo dono delle sei Memorie che Ella si compiacque inviarmi, e alcune delle quali mi riescono in questo momento utilissime. Ella riceverà fra non molto una mia Nota su argomento di elettrostatica, ${ }^{63}$ provocata in parte da una certa discussione che regna da molti anni in Italia sui principii fondamentali di questa scienza, per opera principalmente di Volpicelli, professore di fisica matematica a Roma. Per verità le obbiezioni di costui non meriterebbero molta attenzione; ma siccome non manca chi se ne lascia preoccupare, così non è forse inutile totalmente il combatterle. ${ }^{64}$ Ma io lo farò senza nominare nessuno, e senza neppure accennare alla controversia.

\footnotetext{
${ }^{59}$ Lipschitz, R., Extrait de six mémoires dans le journal de mathématiques de Borchardt, Bulletin des sciences mathématiques et astronomiques, 4, 1874, p. 97-110, p. 142-157, p. 212-224, p. 297-307, p. 308-320, p. 414-420.

${ }^{60}$ Lipschitz was indeed appointed corresponding member of the Academy of Lincei.

${ }^{61}$ Rudolf Clausius (1822-1888) studied at the universities of Berlin and Halle. In 1855 he became professor of mathematical physics at the new Polytechnicum of Zürich. In 1867 he accepted a professorship at the University of Würzburg and in 1869 moved to the University of Bonn. His main contributions concern heat theory and, in particular, the foundations of thermodynamics.

${ }^{62}$ Wilhelm Joseph von Wasielewski (1822-1896) was a musician. He studied at the Conservatory of Leipzig and then moved to Bonn. He published an important biography of R. Schumann (1858), the volume Die Violine und ihre Meister (1869) and other books.

${ }^{63}$ Beltrami, E., Intorno ad alcune questioni di elettrostatica, Rendiconti del R. Istituto Lombardo, s. $2,10,1877$, p. $171-185$; in [2] 3, p. 73-88.

${ }^{64}$ Paolo Volpicelli (1804-1879) studied philosophy at the University of Rome, where he taught at secondary schools from 1832. In 1872 he was appointed professor of mathematical physics at the University of Rome. He was the author of about 200 publications on number theory, experimental physics, and history of science. In his treatise Analisi $e$ rettificazione di alcuni concetti e di alcune sperienze che appartengono alla elettrostatica (Roma, Tipografia Belle Arti, 1866) Volpicelli explained his ideas on electricity. He was against the "recent" electric theory according to which a unique electric force exists.
} 
Applaudisco di gran cuore al felice pensiero, che Ella ha cominciato ad attuare, di una propedeutica matematica agli studii di fisica razionale. Ella sarà benemerito di questa scienza in doppia guisa, colle ricerche originali, e colle agevolezze procurate a chi deve studiarla, ed io faccio voti perché mi sia dato fruire presto di tale ajuto, e farne fruire l'insegnamento.

Non Le sembra che gioverebbe molto un lavoro analogo, ma in fondo inverso, da farsi da un fisico, cioè l'esposizione dei fenomeni accertati, in forma schematica, senza l'attiraglio störend dei minuti procedimenti sperimentali? Io desidero sempre un tal lavoro. Mi creda sempre

Obbl.mo affez.mo suo

E. Beltrami

\section{Letter n. 4}

\section{Eugenio Beltrami to Rudolph Lipschitz}

Illustre Sig.r Professore

Nel ringraziarLa del recente invio dell'interessante Memoria sul principio di Gauss ${ }^{65}$ mi reco a dovere di avvertirLa che io mi sono trasferito dall'Università di Roma a quella di Pavia, e che quindi è bene ch'Ella mi indirizzi in quest'ultima città le future pubblicazioni che vorrà favorirmi.

Al Sig.r Kaiser, che mi chiese, dietro una di Lei indicazione, una copia della breve nota sulla cinematica pseudosferica, ${ }^{66}$ ho tosto fatto la chiesta spedizione.

Sto qui attendendo ad un corso di lezioni sulla fisica matematica, e precisamente, per quest'anno, sull'elettrostatica. Mi sarebbe molto gradito d'avere le di Lei pregiate Memorie sull'argomento; ${ }^{67}$ e, se mai Ella ne conservasse qualche esemplare, io oserei (fidando nella ben nota cortesia di Lei) di farLene richiesta. Posso bensì consultarle nel Journal der Mathematik, la cui collezione esiste nella Biblioteca dell'Università: ma non avendole in separato esemplare, mi riesce meno facile il trovarle sempre a disposizione.

Mi perdoni, e mi creda sempre

dev.mo aff.mo

E. Beltrami

\section{Letter n. 5 \\ Eugenio Beltrami to Rudolph Lipschitz}

Pavia 20 Giugno 1880

\section{Chiarissimo Sig.r Professore}

Ricevo or ora il 2 volume del Lehrbuch der Analysis, che Ella si è compiaciuto di farmi pervenire. ${ }^{68}$

Sono veramente confuso di tanta Sua gentilezza, alla quale io non posso corrispondere se non col riconoscere tutto il pregio della nuova produzione dovuta alla Sua penna. Mi chiamo ben fortunato di esser messo da Lei, così cortesemente, a immediata cognizione di un libro che sarà premurosamente accolto da tutti gli studiosi, i quali da lungo tempo desiderano un trattato di Calcolo che sia in armonia collo stato attuale della scienza e collo spirito delle più importanti sue applicazioni.

\footnotetext{
${ }^{65}$ Lipschitz, R., Bemerkungen zu dem Princip des kleinsten Zwanges, Journal für die reine und angewandte Mathematik, 82, 1877, p. 316-342.

${ }^{66}$ Beltrami, E., Formules fondamentales de Cinématique dans les espaces de courbure constante, Bulletin des sciences mathématiques et astronomiques, 11, 1876, p. 233-240; in [2] 3, p. 23-29

${ }^{67}$ Lipschitz, R., Beiträge zur Theorie der Vertheilung der statischen und dynamischen Electricität in leitenden Körpern, Journal für die reine und angewandte Mathematik, 58, 1861, p. 1-53; Untersuchungen über die Anwendung eines Abbildungsprincips auf die Theorie der Vertheilung der Electricität, Ibid., 61, 1863, p. 1-21.

${ }^{68}$ Lipschitz, R., Lehrbuch der Analysis. Zweiter Band: Differential-und Integralrechnung, Bonn, Cohen, 1880.
} 
Unicamente per attestarLe in qualsiasi modo la mia gratitudine, Le invio alcune recenti pubblicazioni, che avevo messe da parte per unirLe ad altre di cui aspetto gli esemplari. Voglia gradirLe colla Sua solita bontà e credermi sempre

di Lei Ch.mo Sig.r Professore obbl.mo dev.mo E. Beltrami

\section{Letter n. 6 \\ Eugenio Beltrami to Rudolph Lipschitz}

Roma 18 Marzo 1898

\section{Chiarissimo Collega}

La ringrazio veramente di cuore delle benevole espressioni contenute nella cara Sua lettera del 13 corrente. Esse sono una nuova prova della di Lei buona e cordiale amicizia. Quanto all'onore conferitomi dall'Accademia dei Lincei, io ho dovuto accettarlo per deferenza alla volontà dei Colleghi, ma pur troppo non so se le mie forze saranno proporzionate al bisogno. ${ }^{69}$

Nello scorso anno ebbi un grave attacco di malattia dal quale ho potuto fortunatamente salvarmi, ma non posso dire di trovarmi interamente ristabilito in perfetta salute. Comunque sia io Le presento l'espressione della mia sincera riconoscenza, insieme con quella dei voti che io faccio per la di Lei prosperità.

Dev.mo aff.mo E. Beltrami

\section{Letter n. 7 \\ Eugenio Beltrami to Rudolph Lipschitz}

$$
\text { 13/8/[...] Macugnaga (Monte Rosa) }
$$

Chiarissimo collega

Ricevo qui la di Lei lettera e il primo volume dell'Opera di cui Ella mi scrisse già in addietro. ${ }^{70}$ Le sono oltremodo obbligato del prezioso regalo, che mi propongo di ben presto esaminare e di studiare, e intanto mi affretto a dargliene ricevuta.

Sono qui da una quindicina a fare la vita di montagna. Passerò poi i mesi di Settembre e di Ottobre a Venezia, e colà spero potermi occupare piò utilmente dello studio del di Lei libro.

Mi creda, con devota attenzione, obbl.mo Beltrami

\subsection{Letters by Beltrami to Gösta Mittag-Leffler}

The letters by Eugenio Beltrami to Gösta Mittag-Leffler ${ }^{71}$ are contained in MittagLeffler's Archive, Djursholm, Stockholm.

\footnotetext{
${ }^{69}$ Beltrami was appointed President of the Academy of Lincei.

${ }^{70}$ Lipschitz, R., Lehrbuch der Analysis. Erster Band: Grundlagen der Analysis, Bonn, Cohen, 1877.

${ }^{71}$ Gösta Mittag Leffler (1846-1927) studied at Uppsala, Paris, Göttingen, and Berlin. In 1876 he was appointed professor at the University of Helsinki and, in 1881, moved to the new University of Stockholm. He mostly contributed to complex analysis by following Weierstrass's ideas, but also to analytic geometry and theory of probability. In 1882 he founded and then directed the journal Acta Mathematica. Mittag-Leffler engaged himself in finding a position for the Russian mathematician Sonya Kovalevskaya, who in 1884 obtained a position at the University of Stockolm for five years. Some of these letters are devoted to the case of Sonya Kovalevskaya. See also the Appendices 4.7 and 4.8.
} 
Letter n. 1

Eugenio Beltrami to Gösta Mittag-Leffler

Pavie 8 Janvier 83

Cher Monsieur

J'ai reçu les deux exemplaires du 1er cahier des Acta mathematica et je vous remercie bien vivement de l'honneur que vous avez bien voulu me faire en m'en offrant un. J'ai déjà envoyé l'autre exemplaire à l'Institut de Venise, ${ }^{72}$ avec un article de présentation, qui sera lu par un Membre qui se charge de faire cela en mon nom. Cette communication sera faite dans la premire séance que l'on tiendra, et je vous ferai connaître, à son temps, les détails de cette communication, ainsi que le compte rendu qui en sera donné.

Je vous remercie ainsi de la demande, que vous avez bien voulu me faire, de contribuer par quelque Article à votre Journal, qui sort sous d'excellents auspices. Je me tiens honoré par cette demande et je me propose d'en tenir compte. Dans ce moment je suis excessivement absorbé par le travail de préparation de deux cours que je fais: mais sitôt que je serai un peu plus libre, je n'hésiterai pas à vous adresser quelque Note qui me paraîtrait moins indigne de figurer dans votre Recueil.

J'ai présenté à ma femme vos salutations et tous les deux nous vous félicitons pour votre récent mariage, pour le bonheur duquel nous faisons tous vos voeux. Veuillez présenter nos hommages à votre épouse, en attendant que l'occasion se présente d'en faire la connaissance personnelle. ${ }^{73}$

J'ai toujours votre nom noté parmi ceux des personnes auxquelles je dois ma photographie. Malheureusement ma paresse et le manque d'occasion (car il n'y a pas de bons ateliers photographiques ici) m'ont fait trop négliger ce devoir. Soyez sûr que je m'empresserai de satisfaire à votre obligent désir, aussitôt que je réussirai à me procurer ce qui me manque encore depuis notre entrevue.

Veuillez agréer, cher collègue, l'expression de mon sincère dévouement.

E. B.

\section{Letter n. 2 \\ Eugenio Beltrami to Gösta Mittag-Leffler}

Pavie 10 Mai 1883

Monsieur et honoré collègue

J'ai l'honneur d'expédier à votre adresse, dans un paquet sous bande, quelques exemplaires de la communication par laquelle j'ai annoncé à l'Institut Royal de Venise l'offre, de votre part, du 1er Cahier des Acta. À la suite de cette communication, l'Institut a voté un remerciement accompagné de l'offre de ses Comptes rendus, ${ }^{74}$ en échange de votre Journal. Peut être le Secrétaire de l'Institut vous a déjà donné participation de cette délibération. Dans le cas où la chose souffrait quelque retard, veuillez avoir l'obligeance de me le marquer, afin que je puisse faire des démarches, pour la solliciter.

Je saisis cette occasion pour vous confirmer mes sentiments de considération très-distinguée, pour vous prier de vouloir bien présenter mes hommages à M.me votre épouse et pour me dire

Votre bien dévoué

E. Beltrami

\section{Letter n. 3}

\section{Eugenio Beltrami to Gösta Mittag-Leffler}

\footnotetext{
${ }^{72}$ The Istituto Veneto di Scienze, Lettere ed Arti

${ }^{73}$ In 1882 Mittag-Leffler had married Signe af Lindfors who came from a wealthy family.

${ }^{74}$ Beltrami refers the Atti dell'Istituto Veneto di Scienze, Lettere ed Arti.
} 
Monsieur et honoré collègue

Profitant de vos invitations flatteuses, je prends la liberté de vous envoyer un Article pour votre Journal de Mathématiques. Le manuscrit de cette communication vous parviendra sous-bande recommandée, que je remettrai à la Poste en même temps que la présente. ${ }^{75}$

Vous devez me dire sans scrupule si vous croyez que l'Article en question, à cause de son sujet, puisse entrer convénablement dans le cadre de vos publications.

Dans le cas où il vous paraissait admissible, je désirerais, lorsque le moment sera venu, de pouvoir faire au moins la dernière révision des épreuves. Je n'aurai probablement rien à changer, mais il pourrait m'être échappé quelque impropriété de rédaction en français, ou l'on pourrait n'avoir pas exactement reproduit quelque mot ou quelque formule.

Je resterai ici encore un mois, mais si vous voudrez bien me dire à quelle époque, à peu près, les épreuves pourraient arriver, je pourrai vous indiquer, de mon côté, l'adresse qu'il serait bon d'adopter.

Je Vous prie de vouloir bien présenter mes hommages et ceux de ma femme à Mme Mittag-Leffler et agréer l'expression de ma considération et de mon dévouement.

Votre bien obligé

E. Beltrami

\section{Letter n. 4 \\ Eugenio Beltrami to Gösta Mittag-Leffler}

Pavie 19 Juillet 1883

Très honoré collègue

J'adresse la présente à Stockholm, puisque je pense que, si vous n'y êtes pas revenu, on vous la renverra à votre adresse actuelle, que je n'ai pas bien comprise.

Je vous remercie de l'empressement que vous avez bien voulu mettre à agréer l'article que je vous ai envoyé. Je m'arrangerai de manière à me faire renvoyer les épreuves que vous comptez adresser à Pavie. La chose serait mieux assurée si ces épreuves étaient expédiées en enveloppe fermée (le manuscrit ne m'est point nécessaire), car j'ai l'habitude de faire retenir ici tous les imprimés qui arrivent sous bande ouverte, à cause des chances d'égarement qu'il y a lorsqu'on se déplace souvent, comme je fais pendant les vacances. Mais en tous cas j'ai ici un ami qui veut bien se charger de passer en revue ce qui arrivera à mon adresse (car ma maison reste vide).

Si cela n'est pas en dehors des règles que vous avez établi pour le tirage à part, je serais bien aise d'avoir au moins 50 exemplaires de ma Note, car je voudrais bien en faire connaître le contenu à ceux qui s'occupent du sujet. Il est inutile d'ajouter que je tiens à être informé de toutes les dépenses qui seront nécessaires soit pour le tirage, soit pour l'envoi du paquet des exemplaires séparés, soit pour celui des épreuves, notamment si vous consentez à le faire par lettre fermée. En vous renvoyant les épreuves, je vous écrirai pour vous indiquer le lieu où il me serait le plus agréable de recevoir les exemplaires.

Je vais partir dans trois ou quatre jours pour la Suisse, mais je ne peux vous indiquer exactement, dans ce moment, le lieu où je me fixerai. Si, dans une semaine, je pourrai prévoir de rester longtemps dans le même endroit, je vous ferai connaître mon adresse, et alors il n'y aura plus la difficulté dont je vous parlais tout à l'heure.

Il est très-probable que M. Casorati ${ }^{76}$ sera avec moi, en Suisse. Dans ce moment il ne se porte pas tout-à-fait bien, mais j'espère qu'il ne s'agisse que d'une indisposition passagère. Je vous fais ses salutations les plus cordiales.

\footnotetext{
${ }^{75}$ Beltrami refers to his paper later published in Acta: Sur les couches de niveau électromagntique, Acta Mathematica, 3, 1884, p. 141-152; in [2] 4, p. 77-86.

${ }^{76}$ Felice Casorati (1835-1890) was professor at the University of Pavia. He mainly studied real and complex analysis.
} 
J'espère que vous aussi vous pensez, quelque autre fois, à venir passer quelque temps en Suisse avec M.me votre femme. Vous aurez là l'occasion de rencontrer plusieurs professeurs, notamment de l'Allemagne. Il y a quelques annes, j'ai pu jouir pendant plusieurs jours de la compagnie de MM. Zeuthen, Frobenius, Hirst, Schläfli, Geiser, Rosanes, ${ }^{77}$ qui se trouvaient tous en même temps dans le village où je m'étais établi avec MM. Cremona ${ }^{78}$ et Casorati. L'année passée, aussi, étant avec M. Cremona à Pontresina, nous y avons trouvé MM. Kronecker et Helmholtz, avec leurs familles. ${ }^{79}$

Veuillez agréer mes salutations cordiales ainsi que celles de ma femme, et n'oubliez pas de présenter nos hommages à M.me votre épouse, si elle est avec vous en ce moment.

C'est toujours avec la plus grande estime que je vous prie de me croire Votre bien obligé et dévoué

E. Beltrami

\section{Letter n. 5}

\section{Eugenio Beltrami to Gösta Mittag-Leffler}

Silvaplana (Engadina) 25 Juillet 83

\section{Monsieur}

Arrivé ici ce matin, et ayant rencontré des conditions de logement assez convenable, je puis vous dire que je resterai ici au moins trois semaines. Par suite, vous pouvez donner l'ordre qu'on m'adresse ici les épreuves que je vous savez, par envoi ordinaire sous-bande (Drucksache): ainsi aucun retard n'aura lieu pour le retour de ces épreuves après ma correction. J'attends M. Casorati demain ou après demain: je vous avance ses compliments en même temps que je Vous renouvelle mes salutations les plus cordiales.

Votre bien obligé E. Beltrami

\section{Letter n. 5 Eugenio Beltrami to Gösta Mittag-Leffler}

Monsieur et excellent collègue

J'ai reçu et renvoyé à M.r Eneström, il y a quelque jours, les épreuves de ma Note pour les Acta, et, après avoir remercié M.r Eneström pour son obligeance et pour ses soins, je ne veux pas tarder

\footnotetext{
${ }^{77}$ Hieronymus Georg Zeuthen (1839-1920) studied mathematics at the University of Copenhagen, where he was appointed professor in 1871. His main research topics were algebraic geometry and history of mathematics. Georg Ferdinand Frobenius (1849-1917) was one of Weierstrass' students at the University of Berlin. He taught at the University of Berlin and at the Polytechnic of Zürich. His research concerns group theory and the theory of differential equations. Thomas Archer Hirst (1830-1892) was born in England, studied mathematics and physics in Germany and then lived in Paris and Rome. In 1860 he came back to England were he started his academic career in London. He published many papers and book on geometry, algebraic geometry, and physics (mechanics and thermodynamics). Ludwig Schläfli (1814-1895) studied theology at the University of Bern, and then accepted a post as a teacher of mathematics and science at secondary school in Thun. In 1848 he became Privatdozent at Bern, in 1853 extraordinary professor and in 1868 full professor. His works concern $n$-dimensional geometry and invariant theory. Carl Friedrich Geiser (1843-1934) studied and taught at the Polytechnic of Zürich. His research concerns algebraic geometry, differential geometry and invariant theory. Jakob Rosanes (1842-1922) studied mathematics and physics at the University of Breslau, where he taught from 1873 onwards. His main research subjects were algebraic geometry and invariant theory.

${ }^{78}$ Luigi Cremona (1830-1903) was professor at the University of Bologna, taught at the Polytechnic of Milan, and directed the Scuola degli Ingegneri in Rome. He especially contributed to the theory of surfaces and curves.

${ }^{79}$ Leopold Kronecker (1823-1891) taught at the University of Berlin from 1862 onwards, but achieved an official position only in 1883 when Kummer retired. He mainly contributed to analysis and number theory. Hermann von Helmholtz (1821-1894) was professor of physiology at the universities of Königsberg (1849), Bonn (1855) and Heidelberg (1858). In 1871 he became professor of physics at the University of Berlin. He contributed to medicine, physiology, physics, and mathematics.
} 
plus longtemps à vous remercier vous même pour la promptitude avec laquelle vous avez bien voulu pouvoir à l'impression de la susdite Note.

Comme je quitterai ces lieux dans une semaine pour me rendre à Padoue, après une autre semaine que j'emploierai à faire un petit tour dans la Suisse, j'ai donné mon adresse dans la ville susdite pour l'envoi des exemplaires à part: je vous transcrirai cette adresse ci-dessous, quoique je l'aie déjà indiquée à M.r Eneström.

Je vous fais les salutations amicales de M.r Casorati, qui est ici avec nous, et qui est notablement amélioré dans son état de santé depuis qu'il a quitté Pavie.

Je vous présente aussi, au nom de ma femme et au mien, les hommages les plus sincères pour M.me votre épouse et je vous prie de me croire toujours

Votre bien obligé

E. Beltrami

Adresse pour les exemplaires à part:

Sig. Professore Eugenio Beltrami

Padova (Italia) Via S. Fermo 1251

\section{Letter n. 7 \\ Eugenio Beltrami to Gösta Mittag-Leffler}

Venise 2 bre 83

Monsieur et très honoré collègue

Arrivé à Padoue après une absence assez longue, j'ai retrouvé là, en bon ordre, le paquet des exemplaires à part de la Note que vous avez eu la bonté d'accueillir dans les Acta.

Je saisis cette occasion pour vous remercier encore une fois de toutes vos bontés et de tous vos soins et pour vous prier de vouloir bien exprimer ma reconnaissance à M. Eneström pour toutes les peines qu'il a eues à cause de moi.

J'ai à vous prier de vouloir bien me faire connaître le montant de ma dette envers l'Administration des Acta, pour le tirage à part et pour les frais de poste et autres. Je ne reviendrai définitivement à Pavie que dans un mois, mais, comme il est probable que je devrai me déplacer dans l'intervalle, il est bon de m'adresser toujours les lettres à Pavie, d'où on me les renvoie là où je me trouve.

Veuillez présenter mes compliments les plus respectueux à Madame votre épouse et agréer l'expression de mon affectueux dévouement.

Votre bien obligé E. Beltrami

\section{Letter n. 8}

\section{Eugenio Beltrami to Gösta Mittag-Leffler}

Venise 18 bre 83

\section{Cher Monsieur}

Je vous remercie infiniment du portrait d'Abel dont vous avez voulu me faire cadeau. A cause d'une erreur de la Poste il avait d'abord été renvoyé à une autre personne de mon nom, mais fort heureusement il est revenu hier dans mes mains.

Je n'insiste pas, d'après ce que vous avez l'obligeance de m'écrire, dans ma demande. Quant à votre désir bienveillant, vous pouvez être assuré que je tiendrai à honneur de vous envoyer quelque article, lorsque je penserai qu'il puisse paraitre dans les Acta sans faire de tort à la réputation de ce nouveau Recueil.

Ma femme et moi nous remercions M.r et M.me Mittag Leffler de leur bon souvenir et présentons leur nos salutations les plus distinguées. 
Votre bien dévoué

E. Beltrami

\section{Letter n. 9 \\ Eugenio Beltrami to Gösta Mittag-Leffler}

Pavie 17 Mars 1889

\section{Cher collègue et ami}

Au retour d'une courte absence, qui a été motivée par des affaires de famille, je viens de recevoir votre aimable lettre du 11 de ce mois ainsi que le Mémoire de M.me Kowalewsky, que vous avez l'obligeance de m'envoyer et que je reçois avec le plus grand plaisir. ${ }^{80}$ J'étais très-désireux de savoir comment cette excellente géomètre avait pu remporter de nouvelles victoires sur un domaine qui avait été le champ d'aussi grandes batailles analytiques, et je vient de reconnaître, d'une manière encore très-sommaire, qu'il s'agit d'un exploit des plus originels et de plus brillants. Aussi vous faisje mes compliments d'avoir, en bon Rédacteur en chef, assuré aux Acta la prémisse de cet admirable travail.

Il y a une discontinuit très-extraordinaire à vous parler, après ça, de l'invitation bienveillante que vous me faites de vous envoyer, pour plus tard, quelque Article pour les Acta; mais il me [...] mal de ne pas vous en remercier, de tout mon coeur, et de vous assurer que je ferai mon possible pour vous satisfaire, si je réussirai à vous offrir quelque chose qui me semble peu digne de vous et de votre Journal.

Je dois vous remercier de même de l'intention excessivement obligeante que vous avez de m'envoyer le grand mémoire couronné de M. Poincaré, ${ }^{81}$ dont les travaux acquièrent une encore plus grande importance, pour moi en particulier, depuis que leur Auteur se rapproche de plus en plus au domaine de la physique mathématique.

Vos renseignements au sujet de mon allée à Rome ne sont pas tout-à-fait exacts. Il ne s'agit pas de la chaire d'analyse supérieure, qui d'ailleurs a toujours existé, peut-on dire, car elle a été occupée pendant plusieurs années par M. Battaglini, et l'est maintenant par un suppléant: il s'agissait de celle de physique mathématique, qui est vacante depuis plus de dix ans (savoir après la mort de Volpicelli, l'ancien secrétaire perpétuel de l'Académie des Lincei qui lui doit de ne pas avoir été tout à fait supprimée par le Pape). Je n'ai pas absolument renoncé à occuper cette position et si je ne m'y suis pas encore décidé c'est, indépendamment de quelques circonstances de famille, parce que je ne crois pas que je ferais grande chose, à moi-seul, c'est-à-dire sans des cours bien et fortement organisés sur les autres parties des hautes mathématiques. Tout cela serait bien facile si M. Cremona revint aux études avec l'ardeur d'autrefois: mais malheureusement je ne puis pas y compter.

Ma femme vous est bien obligée de vos compliments et nous vous prions tous les deux de vouloir bien présenter les nôtres à M.me Mittag-Leffler. M.r Casorati aussi Vous fait ses salutations affectueuses: je voudrais pouvoir vous dire que sa santé est bonne, mais peut-être laisse-t-elle quelque chose à désirer, quoique il ne s'en plaigne pas: probablement, après le mariage de sa seconde fille, il n'a pas encore trouvé un système de vie qui lui convienne. Veuillez agréer, très-cher ami, mes salutations les plus dévouées.

E. Beltrami

Letter n. 10

\section{Eugenio Beltrami to Gösta Mittag-Leffler}

\footnotetext{
${ }^{80}$ Sonya Kovalevskaya (1850-1891), pupil of Weierstrass, became extraordinary professor of mathematics at the University of Stockholm in 1884 and ordinary professor at the same university in 1889 . In 1888 she won the Prix Bordin (proposed by the Academy of Sciences of Paris) with the paper Sur le problème de la rotation d'un corps solide autour d'un point fixe, Acta Mathematica, 12, 1889, p. 177-232. Most probably Beltrami refers to this paper.

${ }^{81}$ Beltrami refers to the work by Henri Poincaré (1854-1912) - awarded by the King of Sweden Oscar II on January 21st, 1889 - Sur le problème des trois corps et les équations de la dynamique, Acta Mathematica, 13, 1890, p. 1-278.
} 
Mon cher ami

Je viens de recevoir votre aimable lettre et j'aime à vous répondre tout de suite, soit pour vous assurer de la réception de vos envois, soit pour vous dire que je vais me mettre tout de suite à préparer ma réponse à la lettre officielle de l'Université. Je vous suis bien obligé du témoignage d'estime que vous m'avez procuré et que je serais bien heureux de mériter: mais je ne puis vous cacher ma conviction que M. Betti ou M. Brioschi ${ }^{82}$ auraient pu formuler un jugement bien plus autorisé que le mien. Quoiqu'il en soit ce sera pour moi un plaisir que de contribuer à une cause aussi juste que celle de mettre M.me Kowalewsky à l'abri des attaques des personnes malveillantes dont vous me parlez.

Je saisis aussi cette occasion pour vous offrir la photographie que vous m'aviez demandé depuis si longtemps: elle n'est pas d'un aussi bon travail que celle que vous avez bien voulu m'envoyer, et dont je vous remercie bien vivement, mais il me semble que la ressemblance soit assez bien gardée.

Je ferai au plus tôt votre commission à M.r Casorati, que je ne vois depuis quelques jours et que je crois toujours plongé dans certaines recherches sur la courbure des surfaces dont il m'a parlé la dernière fois que je l'ai vu, et dont il était profondément intéressé. Peut-être même la proposition qu'il vous a fait a-t-elle rapport avec ce sujet: mais il ne m'en a rien dit. Je crois que je le verrai demain.

Croyez vous bien de faire aussi vite le voyage de Paris, pour visiter l'Exposition? ${ }^{83}$ D'après les correspondances qu'envoi à Notre journal le mieux informé, la Perseveranza, ${ }^{84}$ un très-habile et trèsintelligent correspondant, qui demeure à Paris depuis 30 ans, il résulterait qu'il faut encore un bon mois avant que l'Exposition puisse être considérée comme suffisamment complète: ce correspondant conseille décidemment ses lecteurs à attendre le Juillet. Il est vrai qu'on aurait alors l'inconvénient du chaud.

Je pense que je pourrai vous adresser la lettre pour l'Université après demain, au plus tard: ainsi vous la recevrez sans doute avant votre départ.

Je joins à mes salutations très-affectueuses celles que ma femme me charge de vous faire, et celles que moi même je vous prie de vouloir bien présenter à M.me Mittag-Leffler. Je vous souhaite un bon voyage et je désire aussi qu'il soit profitable pour Votre santé.

Votre bien dévoué et affectionné Eugène Beltrami

P. S. Il me vient à l'esprit que M.me Kowalewsky pouvait bien essayer de voir si le nouveau cas d'intégration qu'Elle a découvert pour le problème du corps tournant, ne trouverait pas aussi une interprétation élégante dans la théorie de la tige élastique, d'après le rapprochement signalé par Kirchhoff (Mechanik, XXVIII Vorles. §6) entre les deux questions. ${ }^{85}$ Mais ce n'est qu'une idée, laquelle d'ailleurs s'est peut-être déjà présentée à cette Analyste distinguée.

E. B.

\section{Letter n. 11}

\section{Eugenio Beltrami to Gösta Mittag-Leffler}

\footnotetext{
${ }^{82}$ Enrico Betti (1823-1892) was professor at the University of Pisa from 1857 onwards. He gave important contributions to algebra, differential geometry and mathematical physics. Francesco Brioschi (1824-1897) was professor at the University of Pavia. He founded (1863) and directed the Polytechnic of Milan. He contributed to the theory of determinants and to analysis.

${ }^{83}$ Beltrami refers to the Exposition Universelle held in 1889 in Paris.

${ }^{84}$ Perseveranza was an Italian journal founded at the eve of the Unification of Italy (1859).

${ }^{85}$ Kirchhoff, G., Vorlesungen über mathematischen Physik. Mechanik, Lepzig, Teubner, 1876. Gustav Robert Kirchhoff (1824-1887) studied at the University of Königsberg, where he had Jacobi and Franz Neumann as teachers. In 1850 he was appointed professor at the University of Breslau. In 1854 he moved to the University of Heidelberg and in 1875 accepted the chair of theoretical physics in Berlin. He contributed to the fundamental understanding of electrical circuits and spectroscopy.
} 
Mon cher collègue

Je vous écris deux mots pour vous dire plusieurs choses. D'abord que j'ai déjà remis à la Poste, en lettre recommandée, ce matin de bonne heure, le Rapport pour l'Université. Naturellement je l'ai adressé à votre nom. Je crains maintenant qu'il ne soit trop long: mais je ne pouvais me dépouiller des mes habitudes rapporteuses.

Ensuite je vous demande bien excuse dans le cas où j'aurais oublié de signer la photographie que je vous ai adressée. Si j'ai été coupable de cet oubli, j'y remédierai à la première occasion où je pourrai vous en envoyer une autre, meilleure.

Enfin je vous prie de me dire si je dois renvoyer à Stockholm la Note des Astr. Nachr. de Mad.me Kowalewsky. ${ }^{86}$ Il me serait sans doute très-agréable de pouvoir bien la garder, mais il peut se faire que l'Autrice n'aie pas à sa disposition d'autres exemplaires. Ainsi j'attends vos indications.

M. Casorati m'a dit que vous lui avez écrit directement et qu'il va vous répondre, ou envoyer la traduction dont il s'agissait.

Avec mille salutations cordiales

E. Beltrami

\section{Enclosed letter by Eugenio Beltrami to Sophia Kovalevskaya}

Madame

À mon retour d'une absence de Pavie, je viens de retrouver ici l'exemplaire, que vous avez eu la complaisance de me faire parvenir, d'un Mémoire où j'entrevois un complément analytique de votre grand travail couronné. ${ }^{87}$ Je m'empresse de vous offrir l'expression de ma reconnaissance bien sincère pour ce précieux cadeau, et je vous demande pardon si je ne pourrai pas de sitôt vous prier d'agréer quelque publication nouvelle. J'ai bien des matériaux assez étendus: mais les préoccupations cruelles auxquelles je suis en proie depuis plusieurs mois, à cause de la santé de ma femme, m'ôtent la calme et la tranquillité d'esprit dont j'aurais besoin pour les mettre en ordre et les rédiger d'une manière convenable.

Veuillez agréer, Madame et très-honorée Collègue, l'expression de mes sentiments de considération la plus distinguée.

E. Beltrami

\section{Letter n. 12 \\ Eugenio Beltrami to Gösta Mittag-Leffler}

Pavie 21 Juin 1890

Monsieur et collègue très-honoré

J'ai reçu votre nouveau Mémoire sur la représentation analytique des intégrales et des invariants des éq.ns diff.les et je vous présente mes meilleurs remerciements pour ce joli cadeau. J'ai pu déjà apprécier, par une première lecture (ce qui est encore bien loin d'une première étude), toute l'importance et tout l'intérêt des vos recherches, dont le point de départ est une représentation conforme qui rentre dans un type ayant déjà rendu des services considérables en physique mathématique.

\footnotetext{
${ }^{86}$ Kovalevskaya, S., Zusätze und Bemerkungen zu Laplace's Untersuchung über die Gestalt der Saturnringe, Astronomische Nachrichten, 111, 1885, p. 37-48.

${ }^{87}$ Beltrami refers to the paper: Kovalevskaya, S., Sur une propriété du système d'équations différentielles qui définit la rotation d'un corps solide autour d'un point fixe, Acta Mathematica, 14, 1890-91, p. 81-93. This paper is a complement of the memoir cited in footnote 80 .
} 
Je regrette de ne rien avoir à vous offrir de mon côté. Cette année a été excessivement peu favorable à mes études. Une longue maladie que ma femme a eu cet hiver et dont elle est encore bien loin d'être guérie aussi complètement qu'il y avait bien de l'espérer, m'a profondément troublé et c'est à peine si j'ai eu la force de m'occuper de mes leçons à l'université.

Ce n'est pas, aussi, un petit chagrin pour moi que celui d'assister, sans ne pouvoir rien faire, à la lente destruction de la santé de mon excellente ami M. Casorati. Il vous aura peut-être écrit lui-même de se trouver incommodé depuis quelque temps: mais il s'est toujours fait, et il se fait encore, une illusion complète sur son état. Il est, depuis quelques jours, à la campagne, où il désirait vivement d'aller, et pour où on l'a laissé partir, vu qu'il n'y avait absolument rien à faire pour lui. Il est victime d'une affection cancéreuse aux intestins, maladie terrible dont sont morts son père et sa mère. Qui sait si je pourrai le revoir encore avant que je part pour les vacances; puisque, dans la sécurité où il vit, ma visite de ma part lui semblerait extraordinaire et pourrait lui réveiller des soupçons! Vous me croirez facilement si je vous dis que tout cela me cause une grande douleur.

Je vous prie d'agréer, pour vous et pour votre honorable famille, l'expression de mes voeux sincères pour votre bonheur aussi que celle de ma considération bien distinguée et de mon affectueux dévouement.

E. Beltrami

\section{Letter n. 13}

\section{Eugenio Beltrami to Gösta Mittag-Leffler}

Pavie 25 Novembre 1890

Monsieur et collègue très-honoré

Je viens vous présenter mes remerciements les plus empressées, pour la bonté que vous avez eue de me faire parvenir une exemplaire du grand Mémoire couronné de M. Poincaré. Si je suis un peu en retard avec vous pour cet objet, c'est que j'ai dû m'absenter dans ces derniers jours, soit pour prendre part aux élections politiques, soit pour satisfaire au désir de mon vieil et excellent ami M. Cremona, qui m'avait prié d'assister, en qualité de témoin pour la fiancée, au mariage de sa dernière fille avec un médecin distingué de Naples.

Je ne puis juger jusqu'à présent de l'importance et du contenu du Mémoire de M. Poincaré que d'après la préface et la table des matières, ce qui suffit cependant à prévoir qu'il s'agit d'un travail fondamental; comme vous me l'aviez déjà assuré, au reste, à la suite de la lecture que vous aviez faite de la rédaction primitive. Mais je vais en prendre une connaissance plus détaillée, au moins en tant que cela me sera permis par mes occupations obligatoires, qui sont plus absorbantes au commencement des cours que dans la suite.

Je suis encore sous le coup du malheur qui nous a frappés, dans la personne de notre excellent ami M. Casorati. M. Brioschi est occupé à écrire une commémoration scientifique de ce savant, qui a été d'abord son élève et puis son ami, peut-être le plus intime. Cet écrit paraîtra, je crois, dans les Annali di Matematica et comprendra la liste complète des articles et des Mémoires publiés par M. Casorati. ${ }^{88}$ Il pourrait vous être très-utile dans le cas où vous voudriez consacrer quelques pages des Acta au souvenir d'un de ses collaborateurs étrangers. ${ }^{89}$ La place que M. Casorati a laissé vacante ici va être occupée par un jeune analyste très-distingué, M. Ernest Pascal, ${ }^{90}$ qui a fait tout récemment une série de travaux sur les fonctions hyperelliptiques.

J'ai passé une bonne partie des vacances en Tyrol, à cause d'un traitement minéral que M.me Beltrami devait suivre pour améliorer son état de santé. Cet état est maintenant assez satisfaisant: mais je ne suis pas sans craintes à cause de l'hiver, qui a été très-doux jusqu'ici, mais qui devient

\footnotetext{
${ }^{88}$ In the volume 18 (1890) of Annali di matematica pura ed applicata Brioschi shortly communicated the death of Felice Casorati and promised to publish Casorati's obituary in the next volumes of Annali, but it never appeared.

${ }^{89}$ In Acta Mathematica no obituary of Felice Casorati has been published.

${ }^{90}$ Ernesto Pascal (1865-1940) studied at the universities of Naples, Pisa and Göttingen with Klein. In 1890 he was appointed professor of calculus at the University of Pavia. In 1907 he move to the University of Naples. His works deal with various research fields; in differential geometry he developed a kind of generalization of Ricci's tensor calculus which, however, did not lead to any interesting consequence.
} 
d'ordinaire très-rigide dans les mois de Décembre et de Janvier. C'est la saison la plus défavorable à la santé de mon épouse et celle qui exige le plus de précautions.

Je vous prie de vouloir bien, cher collègue et ami, présenter mes compliments les plus distingués a votre honorable famille, ainsi qu'à votre beau-frère M.r Del Pezzo, ${ }^{91}$ s'il habite encore Stockholm en ce moment: veuillez bien aussi recevoir mes salutations les plus affectueuses, ainsi que l'expression renouvelée de ma reconnaissance.

Votre bien obligé E. Beltrami

\section{Letter n. 14 \\ Eugenio Beltrami to Gösta Mittag-Leffler}

Pavie 28 Décembre 1890

Cher et honoré collègue

J'ai beaucoup retardé à répondre à votre bonne lettre du 26 du mois passé (qui s'est croisée avec celle que je vous écrivais pour vous remercier de l'envoi du grand Mémoire couronné de M. Poincaré), parce que j'étais très-contrarié de ne pas pouvoir satisfaire a votre demande d'un exemplaire à part de mes écrits sur l'hydrodynamique. ${ }^{92}$ Je m'en trouvais en effet tout-à-fait dépourvu, le tirage en ayant été assez faible. Cependant je me suis adressé à plusieurs de mes amis pour voir si quelqu'un d'entre eux, pour qui le sujet de ces Mémoires n'avait pas un grand intérêt, consentirait à m'en faire la restitution; et j'ai enfin obtenu de l'un d'eux la promesse de me les renvoyer. J'en ferai relier les feuilles en brochure, avec le frontispice dont je conserve encore quelques tirages, et je vous en ferai l'envoi dans quelques jours, en y joignant, conformément à votre désir, le peu d'autres ancien articles que je pourrai retrouver. Je dois vous dire, encore à propos des mémoires sur l'hydrodynamique, qu'à présent je suis très-peu content de ce travail, qui a paru nécessairement par fragments, et dont le plan s'est pour ainsi dire changé dans le cours de la rédaction, ainsi que l'indique assez la différence du titre que j'ai fini par lui assigner, d'avec celui des volumes de l'Académie de Bologne. J'ai eu aussi l'occasion de constater plusieurs erreurs d'impression, plusieurs inadvertances, et même quelques inexactitudes: je me repens, en outre, d'avoir traîné en longueur plusieurs recherches de peu d'importance. Mais, enfin, péché confessé, dit-on, est à moitié pardonné.

Je Vous remercie bien de l'intérêt que vous prenez à la santé de M.me Beltrami. Le Dieu merci, je suis assez content de ce côté: malgré la rigueur du froid de cet hiver, jusqu'à présent il ne s'est produit aucun phénomène grave comme ceux de l'hiver passé et je commence à espérer que la mauvaise saison puisse s'écouler sans accident.

Je vous prie de vouloir bien présenter mes hommages les plus respectueux à M.me Kowalewsky. Je vous prie aussi de vouloir bien agréer, pour vous et pour M.me Mittag-Leffler, les souhaits les plus sincères que M.me Beltrami et moi nous faisons pour votre bonheur, dans la nouvelle année qui va commencer.

Veuillez accueillir l'expression de mes sentiments de considération la plus distinguée. Votre bien dévoué

E. Beltrami

\section{Letter n. 15}

\section{Eugenio Beltrami to Gösta Mittag-Leffler}

\footnotetext{
${ }^{91}$ Pasquale del Pezzo (1859-1936) came from a noble family of Naples. He studied mathematics at the University of Naples, where he was appointed professor of projective geometry in 1884. He married the writer Anne Charlotte Leffler, sister of the mathematician Gösta. Del Pezzo was many times chancellor of the University of Naples, mayor of the town and senator of Italy. He introduced the Del Pezzo surfaces, well-known in modern algebraic geometry, as the surfaces having elliptic curves as plane sections.

${ }^{92}$ Beltrami, E., Sui principi fondamentali dell'idrodinamica razionale, Memorie della R. Accademia delle Scienze dell'Istituto di Bologna, s. 3, 1, 1871, p. 431-476; 2 (1872), 381-437; 3 (1873), 349-407; 5 (1874), 443-484; these works are reprinted in [2] 2, p. 202-379 with the title Ricerche sulla cinematica dei fluidi.
} 
Collègue très honoré

Après un bien long retard, je viens satisfaire au désir par vous exprimé d'avoir un exemplaire de mon Hydrodynamique. Je n'en avais plus chez moi, comme je vous l'ai dit, et je comptais m'en procurer un chez un ami: mais celui que j'ai reçu était si délabré que je n'ai pas osé vous l'offrir. Aussi ai-je dû faire d'autres recherches et j'en ai enfin trouvé un en bon état de conservation: c'est celui que je vous adresse, après y avoir fait quelques corrections à la plume. Il y en aurait bien d'autres à faire; mais, ainsi que j'ai déjà eu l'occasion de vous l'écrire, c'est un ancien travail qu'il faudrait revoir et refaire de fond en comble.

Vous désiriez aussi des exemplaires d'autres anciens Mémoires de moi et j'ai toujours toute la meilleure volonté de vous contenter. Mais les circonstances sont souvent plus fortes que nos volontés. J'ai passé une très-longue période d'appréhensions et de souffrances à cause de la santé toujours imparfaite de M.me Beltrami, et je n'ai eu la tête à rien. A présent les choses vont en peu mieux, mais il surgit des préoccupations d'un autre genre. Il s'agirait pour moi de me transférer à Rome (je crois que vous m'avez parlé une fois de cela, n'approuvant pas ma rénitence à me décider à ce passage): c'est un grand tracas qui va me frapper, mais auquel je pourrai me d'aider en vue de transporter M.me Beltrami en un climat plus doux. La chose n'est pas tout-à-fait décidée, mais elle se décidera assez vite. A cette occasion je devrai faire une grande révision de tous mes papiers et de tous mes livres, et je crois que je vais retrouver beaucoup de choses qui depuis bien longtemps ont disparu ou se sont soustraites à mes recherches. J'espère alors pouvoir former un paquet pour vous, beaucoup moins incomplet que je ne pourrais le faire à présent.

J'ai été énormément affligé de la nouvelle, tout-à-fait imprévue pour moi, de la mort de M.me Kowalewsky. C'est un bien grande perte pour les sciences mathématiques, et pour vous en particulier qui étiez à même d'apprécier de tout fait le mérite exceptionnel de cette femme illustre. J'espère qu'on en publiera une biographie détaillée dans les Acta, afin que les savants de tous pays apprennent les circonstances parmi lesquelles s'est éclore et s'est développée cette intelligence si élevée et si fine, que l'on aurait cru destinée à une bien plus longue carrière! ${ }^{93}$

Veuillez, Monsieur et excellent Collègue, agréer pour vous-même et pour Mad.me votre épouse l'expression de mes sentiments les plus distingués, ainsi que l'assurance de mon parfait dévouement.

E. Beltrami

\section{Letter n. 16 \\ Eugenio Beltrami to Gösta Mittag-Leffler}

Rome 27 Juin 1892

Monsieur et excellent collègue

Je m'empresse de vous adresser ci-jointe la Circulaire, que vous avez bien voulu me transmettre après y avoir apposé une signature et mon adresse. Je suis tout-à-fait heureux de m'associer à la fête que l'on veut faire à l'éminent analyste et je regrette seulement de ne pas pouvoir, à cause de mon prochain départ pour les vacances, contribuer autrement que par une offre personnel. Aussi suis-je bien content que M.r Guccia, ${ }^{94}$ dont l'activité m'est bien connue, soit disposé à prendre sur lui la plus grande partie de la peine. Je vais me mettre en rapport avec lui à cet objet.

Comme vous voyez, je me suis enfin décidé à venir à Rome, où j'ai déjà fait l'année scolaire 91-92. Malheureusement avant que j'eusse eu le temps de m'installer convénablement dans cette ville, M.me Beltrami a été attaquée par l'influence, à la suite de la quelle elle a eu une longue série

\footnotetext{
${ }^{93}$ Mittag-Leffler, G., "Sophie Kowalewsky. Notice biografique", Acta Mathematica, 16, 1892-93, p. 385-392.

${ }^{94}$ Giovan Batti Guccia (1855-1914) studied at the University of Rome, where Luigi Cremona directed his first research in geometry. He was appointed professor at the University of Palermo and in 1884 founded the Circolo Matematico di Palermo. This institution became more and more important in Italy and abroad. The journal of the Mathematical Circle, the Rendiconti del Circolo Matematico di Palermo, was an international mathematical journal which published fundamental papers in the history of mathematics. See [14].
} 
de souffrances qui ne sont pas même aujourd'hui tout-à-fait disparues, et qui nous ont obligé de vivre ici en touristes. Ce n'est qu'en Septembre que nous pourrons faire le déménagement complet. J'espère pouvoir alors reprendre mon travail plus régulièrement, et réparer au temps perdu. Nous espérons aussi, M.me Beltrami et moi, que le jour viendra où nous vous reverrons à Rome, ce qui sera un grand plaisir pour nous. Veuillez agréer, en attendant, nos salutations les plus cordiales ainsi que l'expression des sentiments de profonde et affectueuse estime de

Votre bien dévoué E. Beltrami

This letter is enclosed

Circolare per l'omaggio a Hermite

Palermo, 26/6/1892

Monsieur

Dans quelques mois, l'un des plus éminents géomètres qu'il y ait jamais eu, M. Hermite, va avoir 70 ans. La vie tout entière a été consacrée à la Science. Depuis ces précoces travaux qui attiraient sur un jeune écolier, l'attention de Jacobi, jusqu'à son récent mémoire sur le propriétés du nombre e, il a sans cesse marché de découverte en découverte. De tous ces efforts il se croyait assez récompensé par les progrès de ses deux sciences de prédilection l'arithmétique et l'Analyse et il ne recherchait ni les honneurs, ni la gloire.

Mais s'il fut une notoriété bruyante, il ne reposera pas sans doute un témoignage sincère de reconnaissance et de respect. C'est, pourquoi un groupe d'élèves et d'admirateurs de M. Hermite croit devoir faire appel à ceux qui ont suivi ses leçons, comme à ceux qui l'ont approché ou qu'ont d'une manière quelconque subi son influence. Et nous en effet, nous lui devons beaucoup; non seulement sa parole, ses ouvrages et ses conseils ont guidé nos premiers pas, mais sa vie nous a donné un grande exemple; elle nous a appris à aimer la science d'un amour désintéressé.

Puisse notre concours lui prouver que cette leçon n'a pas été perdue et combler un de ses voeux le plus chers en lui donnant l'espoir que d'autres recolleront un jour, au profit de l'Analyse, la moisson qu'il a si libéralement semée.

Nous espérons, Monsieur, que Vous êtes dans les mêmes sentiments et que Vous penserez comme nous que le meilleur moyen de prouver à M. Hermite notre respectueuse admiration, c'est de lui offrir à l'occasion de son 70 anniversaire, une médaille commémorative avec une adresse portant les signatures de nombreux amis de la science.

Eugène Beltrami

Professeur de physique mathématique

Rome, Institut Royal de Physique, rue de Panisperna 89

\subsection{Some letters concerning Sonya Kowalewskaia's affair of 1886}

Letter n. 1

Enrico Betti to Luigi Cremona

This unpublished letter is contained in the Istituto Mazziniano di Genova

[Su carta intestata della R. Scuola Normale Superiore]

Pisa 26 Marzo 1886

Caro Cremona

Mi scrive Genocchi ${ }^{95}$ che ha ricevuto una lettera di Hermite colla quale gli chiede di fare adesione insieme con i suoi amici matematici d'Italia a una testimonianza in favore della Sig.ra

\footnotetext{
${ }^{95}$ Angelo Genocchi (1817-1889) was professor at the University of Turin from from 1859 . Peano served as his assistant in 1881-82. He contributed to number theory, series and integral calculus.
} 
Sofia Kowalewski, alla quale hanno fatto già adesione Jordan, Darboux, Appel, Poincaré, Picard et Tisserand. ${ }^{96}$ Genocchi ha già aderito unitamente a D'Ovidio ${ }^{97}$ e prega me a mandare la mia adesione, e a scrive a te, Beltrami, Casorati, etc. per sentire se acconsentite a unirvi a noi.

La ragione di questa testimonianza a favore della Sg. Kowalewski è il rigetto della proposta di nominarla Socia dell'Accademia di Stokholm il quale ha destato mali umori e malevolenza verso di Lei per la quale è stato anche posto in dubbio il suo valore matematico.

Genocchi avrebbe scritto da sè se la cataratta progrediente e altri incomodi di salute non gli rendessero troppo grave scrivere lettere.

Ricevi i più cordiali saluti dal

Tuo aff Amico e Collega

\section{Letter n. 2 \\ Eugenio Beltrami to Enrico Betti \\ Beltrami's letters to Betti are contained in Betti's Archive, Archivio della Scuola Nor- male Superiore di Pisa. They will be published in [25]}

Pavia 28 Marzo 86

\section{Carissimo Betti}

La proposta che tu hai fatto, al Casorati ed a me, a nome di Genocchi, ci ha alquanto imbarazzati.

Sotto qual forma si dovrebbe fare la richiesta adesione? Trattasi di formulare, ciascuno personalmente per proprio conto, una dichiarazione di stima, o trattasi di aderire, in genere, ad una dichiarazione redatta da altri, e di cui non si possa conoscere il tenore? - Questa è una prima questione. Una seconda è quest'altra. Cosa fanno i matematici tedeschi? Sarebbe importante saperlo, perché avendo la signora Kowalewsky studiato in Germania, dev'essere colà conosciuta da molti professori valenti, i quali dovrebbero pei primi sentire la necessità di manifestare la loro opinione, dato che una manifestazione cosiffatta sia opportuna e motivata.

A dire la verità è una faccenda che al Casorati ed a me sembra alquanto delicata. Posto anche che l'opposizione dell'Accademia di Stoccolma sia eccessiva, è pur nondimeno desiderabile che gli scrupoli di quelli Accademici vengano rispettati e che non si eserciti dal di fuori una pressione che, in altro momento ed in altre condizioni, potrebbe essere prodotta con fini meno buoni. D'altronde poniamo che quei signori fossero ostili in genere all'ammissione di donne, e non già a quella della signora Sofia in particolare: come si fa ad imporre loro un'altra opinione? Giacché, sebbene si tratti di affermare genericamente il merito della Signora, è certo che in pratica questo verdetto va a colpire gli Accademici. Il Casorati poi osserva giustamente che, essendo stata creata per la Kowalewsky una cattedra apposita, non può essere mancata a lei l'occasione di farsi conoscere al pubblico studioso come insegnante di elevate dottrine matematiche, cosicché, se il suo insegnamento è realmente buono,

\footnotetext{
${ }^{96}$ On March 19th, 1886, Hermite wrote to Genocchi: "Permettez-moi mon cher Président de vous faire part d'une circonstance qui intéresse Madame de Kowalevski, l'éminente analyste, et dont m'a informé M. Mittag-Leffler. Plusieurs membres de l'Académie des Sciences de Stockholm, auraient désiré qu'elle fût appelée à remplir une place vacante dans cette Académie, mais une opposition forte vive s'est produite contre la proposition d'admettre une femme à siéger, quelque fût d'ailleurs son talent scientifique, et Madame Kowalevski n'a pas été elue. Non seulement elle n'a pas été elue, mais une sorte de malveillance s'est attachée à sa personne, et on a été jusqu'à contester son mérite mathématique. M. Mittag-Leffler me demande de prendre sa défense, et dans cette intention, j'ai obtenu de MM. Camille Jordan, Darboux, Appell, Poincaré, Picard, Tisserand, l'autorisation de joindre au mien leur témoignage en sa faveur. Je viens vous demander, sous les plus expresses réserves de votre convenance, de vous réunir aux géomètres français, et dans le cas où ce ne serait point contraire à votre sentiment, de m'obtenir les adhésions de vos amis mathématiques d'Italie.", in [31], p. 179. Other interesting notices on this subject are in [1], p. 161-163. As it is emphasized in section 3.3, though many mathematicians signed the letter, Sonya Kovalevskaya did not become a member of the Academy. Charles Hermite (1822-1901), Camille Jordan (1838-1922), Gaston Darboux (1842-1917), Paul Appel (1855-1930), Henri Poincaré (1854-1912), and Emile Picard (1856-1941) were important French mathematicians; Félix Tisserand (1845-1896) was an astronomer, who in 1886 held the chair of celestial mechanics at the Sorbonne.

${ }^{97}$ Enrico D'Ovidio (1843-1933) was appointed professor at the University of Turin in 1872 . He contributed to (Euclidean and non-Euclidean) geometry. In 1905 he became senator of the Italian Kingdom.
} 
non è ammissibile che un fatto tanto comune, quale è quello di una cattedra accademica non riuscita, possa scuotere la riputazione che essa ha potuto crearsi per questa via.

Mia moglie ricambia i tuoi cordiali saluti.

Aff.mo tuo E. Beltrami

\section{Letter n. 3}

Luigi Cremona to Enrico Betti

The letters by Cremona to Betti are contained in Betti's Archive, Archivio della Scuola Normale Superiore di Pisa, and published in [24], p. 7-90; the cited letter is on page 89.

[Roma] 2 aprile 1886

\section{Carissimo Betti}

Ho ricevuto la tua del 26 , e ti chieggio scusa se non sono stato più sollecito nel rispondere. Ben volentieri aderisco alla manifestazione o testimonianza in onore della Sig. Kowalewski. Perciò, nel rispondere per tuo conto, ti prego di aggiungere al tuo nome il mio, sebbene la mia adesione abbia ben poco valore in confronto alla tua.

Abbimi sempre per tuo affezionatissimo amico

L. Cremona

\section{Letter n. 4}

\section{Eugenio Beltrami to Enrico Betti}

Pavia 11 Aprile 86

Carissimo Betti

Stando le cose come tu scrivi, il Casorati ed io pensiamo di astenerci dall'interloquire nella nota faccenda Kowalewsky, tanto più che realmente né l'uno, né l'altro abbiamo conoscenza sufficiente dei lavori di lei. Del resto i matematici italiani sono già più che distintamente rappresentati fra gli aderenti alla dichiarazione di stima.

Rilevo oggi dal Fanfulla ${ }^{98}$ che il 18 si raduna la Giunta del Consiglio. Ciò mi spiega come la Commissione del Pinto sia stata convocata per il 19: ma io ci ho risposto che non vado.

Mia moglie ricambia i tuoi saluti ed io ti stringo affettuosamente la mano.

Aff.mo tuo E. Beltrami

\subsection{Beltrami's Report on Sonya Kowalewskaia (1889)}

\section{Eugenio Beltrami to the University of Stockholm} This unpublished Report is contained in Mittag-Leffler's Archive, Djursholm, Stockholm ${ }^{99}$

\footnotetext{
${ }^{98}$ An Italian daily newspaper published from 1870 to 1886

${ }^{99} \mathrm{As}$ is pointed out in section 3.3, Sonya Kovalevskaya was extraordinary professor of mathematics at the University of Stockholm since 1884. Also thanks to Beltrami's report, in 1889 she was appointed ordinary professor at the same university. Also Hermite and the Norwegian physicist and meteorologist Vilhelm Friman Koren Bjerknes (1862-1951) addressed their letters of recommendation to the Academy of Science of Stockholm; see [1], p. 131.
} 
À l'Université de Stockholm

Invité par l'Université de Stockholm à exprimer mon avis sur les travaux scientifiques de Madame Kowalewsky et sur les titres que ces travaux peuvent lui donner pour être nommée professeur ordinaire d'Analyse supérieure en cette Université, je m'empresse de répondre par ce qui suit à cette invitation qui m'honore, en même temps qu'elle me donne l'occasion bien agréable de rendre justice à un véritable mérite.

Les travaux dont il s'agit sont tous des pièces d'analyse la plus élevée et la plus exquise: mais tandis que les deux premiers (la Dissertation inaugurale et le Mémoire inséré au J.M. des Acta Mathematica) se apportent par leurs sujets aux mathématiques pures, les trois autres ont trait à des applications physico-mécanique aussi importantes que difficiles. ${ }^{100}$ M'étant moi-même, depuis quinze ans, presque entièrement dévoué à ce genre de recherches, on me permettra de ne m'arrêter un peu que sur ces derniers travaux, d'autant plus que le temps me manquerait de revoir avec assez d'attention les deux premiers dont cependant je me rappelle parfaitement bien l'appréciation ne peut plus honorable qu'en faisait un de mes confrères les plus éminents et les plus compétents, M.r le Sénateur Betti, de Pise. E d'ailleurs un fait bien avéré que la solution exacte des questions mécaniques exige le plus souvent les ressources les plus cachées de l'analyse, ce qui en fait des sujets très propres à donner la mesure des forces et des aptitudes de ceux qui les attaquent.

Je commencerai par le Mémoire très-considérable qui a paru aux J.M. des Acta Mathematica et qui est intitulé Ueber die Brechung des Lichtes cristallinischen Mitteln. ${ }^{101}$ Quiconque a étudié la théorie de la double réfraction exposée par Lamé a pu voir combien est fatigante et enveloppée sa longue recherche finale sur la possibilité d'un seul centre d'ébranlement, et plus d'un lecteur a du penser que cette recherche n'était peut-être pas placée sur la véritable voie. Mme Kowalevsky a conçu l'idée de trancher la difficulté en entreprenant l'intégration directe des équations différentielles du mouvement de l'éther et, ce que l'on doit encore plus admirer, elle a eu le mérite de réussir de la manière la plus complète dans cette entreprise aussi hardie que compliquée. Elle a pu sans doute s'avantager des procédés que Poisson et Cauchy avaient depuis longtemps fait connaître (pour des questions analogues mais beaucoup plus simples) et que M. Weierstrass avait grandement perfectionnés et préparés, en quelque sorte, pour ce dernier effort: mais il y avait encore bien du chemin à faire, bien des obstacles à vaincre. Mme Kowalewsky a su parcourir la carrière jusqu'au bout, sans jamais renoncer ni à l'élégance des formules, ni à la rigueur des déductions. Ce chapitre important de l'optique mathématique a désormais acquis, par elle, sa forme la plus parfaite, et les conséquences des hypothèses qui lui servent de base pourront être maintenant facilement poussées jusqu'aux derniers détails.

Le Mémoire intitulé Zusätze und Bemerkungen zu Laplace's Untersuchungen ueber die Gestalt der Saturnringe ${ }^{102}$ inséré, d'après la proposition de l'illustre Mr. Gyldon, au J.M. des Astronomyschen Nachrichten est beaucoup moins étendu que le précédent, mais se rapporte à un point de la mécanique céleste qui n'est pas moins important, dans cet autre domaine de la science. Il s'agit des conditions d'équilibre du fluide répandu à la surface d'un anneau tournant autour d'un noyau central. Sous certaines hypothèses très-restrictives, cette question avait été résolue par Laplace, mais par des considérations qui, entre autres choses, ne laissaient pas reconnaître bien nettement le degré d'approximation que l'on devait attribuer, en réalité, au résultat formulé par lui. Mme Kowalewsky a repris toute cette question, pour la traiter d'une manière bien plus large et plus complète ; et par

\footnotetext{
${ }^{100}$ The first two papers which Beltrami refers to are: Kovalevskaya, S., Ueber die Teorie di Partielle Differentialgleichungen. Dissertation, 1874 (also published in Journal für die reine und angewandte Mathematik, 80, 1875, p. 1-32); Ueber die Reduction einer bestimmten Klasse Abel'scher Integrale dritten Ranges auf elliptische Integrale, Acta Mathematica, 4, 1884, p. 393-414. The other three papers concerning mathematical physical subjects - and which Beltrami refers to - are: Kovalevskaya, S., Ueber die Brechung des Lichtes cristallinischen Mitteln, Acta Mathematica, 6, 1885, p. 249-304; Zusaetze und Bemerkungen zu Laplace's Untersuchungen ueber die Gestalt der Saturnringe, Astronomische Nachrichten, 111, 1885, p. 37-48; Sur le problème de la rotation d'un corps solide autour d'un point fixe, Acta Mathematica, 12, 1889, p. 177-232.

${ }^{101}$ See footnote 100 . Beltrami did not realize that Kovalevskay committed a fatal mistake. This mistake was pointed out and corrected by Volterra in the paper: Volterra, V., Sur les vibrations lumineuses dans les milieux biréfringents, Acta Mathematica, 16, 1892, p. 153-215. On this subject see [1].

${ }^{102}$ See footnote 100 .
} 
une analyse très-habile elle est arrivée non seulement à bien fixer ce degré d'approximation, mais elle a donné une méthode générale à l'aide de laquelle on pourrait satisfaire aux conditions du problème avec telle approximation que l'on voudrait. On doit surtout admirer, dans ce morceau, la sûreté par laquelle la savante analyste, ne se laissant pas rebuter par de très-réelles complications de calcul, retrouve toujours son chemin et arrive au but qu'elle s'était proposée. Pour ce qui est du dernier Mémoire Sur la rotation d'un corps pesant autour d'un point fixe, T. 12 des Acta ${ }^{103}$ le jugement que vient d'en prononcer l'Académie des Sciences de Paris me dispenserait tout-à-fait d'en parler. Je ne puis cependant m'empêcher de remarquer qu'il s'agit d'un problème qui a été l'objet d'à peu près un siècle d'efforts, et qui, après cela, n'avait été complètement résolu que dans deux cas, auxquels se rattachent les noms d'Euler et de Lagrange. Les brillants aperçus géométriques de Poinsot, que l'on pouvait croire destinés d'abord à élargir la voie, n'ont malheureusement abouti à rien, en ce qui concerne la véritable difficulté, qui est celle de l'intégration, et ont plutôt laissé craindre que le chemin resterait désormais fermé. Mme Kowalewsky a eu le bonheur de découvrir un nouveau cas, intégrable par des fonctions bien connues. J'ai dit le bonheur, mais ce n'est pas le mot, car tout bonheur passe, tandis que le résultat de Mme Kowalewsky restera, et aura sa place marquée dans les traités de mécanique, après les solutions classiques dont j'ai nommé les auteurs.

Je dois maintenant répondre à la seconde partie de la question qui m’a été adressée et dire si les travaux dont je viens de parler peuvent être considérés comme des titres valables pour une nomination de professeur ordinaire à l'Université. Cette question étant en quelque sorte administrative, ou juridique si l'on veut, plutôt que scientifique, ne peut pas comporter, évidemment, une solution absolue. Ayant toutefois été membre, dans mon pays, d'un grand nombre de Commissions de concours et de promotion, avec mandat de juger les titres des candidats aux différentes chaires de mathématiques supérieures, et ayant aussi été membre, pendant quelques années, du Conseil supérieur de l'Instruction publique, qui exerce sa révision sur les Rapports des Commissions et fait ensuite ses propositions définitives au Ministre, je suis porté pour ma parte à assimiler la question dont il s'agit à une de celles que j'ai eu bien souvent à traiter : je veux dire à l'appréciation des titres pour la promotion d'un professeur extraordinaire (nommé d'année en année par le Ministre) au rang de professeur ordinaire (nommé à vie par le Roi). Or je puis déclarer en toute conscience que dans une occasion semblable, les titres à l'appui étaient de la force de ceux de Mme Kowalewsky, la Commission la plus exigeante et la plus sévère n'hésiterait par un instant à donner une approbation unanime et le Conseil n'hésiterait pas non plus, à ratifier pleinement ce jugement: je puis même ajouter que ce Conseil autoriserait le Ministre à excéder d'une unité le nombre des chaires disponibles, s'il se trouvait que ce nombre fût déjà au complet. En me plaçant ainsi au point de vue de ce qui se passerait en Italie, le seul dont je puis parler avec compétence, il n'y a pas de doute, pour moi, qu'en nommant Mme Kowalewsky à une chaire permanente d'analyse, on ferait non seulement acte de justice mais on satisferait d'une manière splendide aux exigences de l'enseignement supérieure.

Ayant ainsi répondu de mon mieux, mais en toute sincérité, aux questions que l'honorable Université de Stockholm a bien voulu m'adresser, je n'ai plus qu'à exprimer à ses dignes représentants les sentiments de profond respect et de très-vive reconnaissance avec lesquels j'ai l'honneur de me signer

Eugène Beltrami

Professeur à l'Université Royale de Pavie (Italia)

\section{Acknowledgment}

I warmly thank Michèle Audin, Livia Giacardi, Jens Høyrup, and Pietro Nastasi for their precious suggestions and Thomas Archibald for checking my English.

\footnotetext{
${ }^{103}$ See footnote 100.
} 


\section{References}

[1] AUDIN, M : Souvenirs sur Sofia Kovalevskaya, Montrouge, Calvage et Mounet, 2008

[2] BELTRAMI, E : Opere matematiche (ed. by Facoltà di Scienze della R. Università di Roma), 4 vols., Milano, Hoepli, 1902-1920

[3] BELTRAMI, E : Saggio di interpretazione della geometria non euclidea, 1868, in [2] 1, p. 374405; French Transl. Essai d'interprétation de la géométrie non euclidienne, Annales scientifiques de l'Ecole Normale Supérieure, 6, 1869, p. 251-288

[4] BELTRAMI, E : Teoria fondamentale degli spazii di curvatura costante, 1869, in [2] 1, p. 406-429; Frenche Transl. Théorie fondamentale des espaces de courbure constante, Annales scientifiques de l'Ecole Normale Supérieure, 6, 1869, p. 347-377

[5] BELTRAMI, E: Sulle equazioni generali dell'elasticità, 1882, in [2] 3, p. 383-407

[6] BELTRAMI, E : Sull'uso delle coordinate curvilinee nelle teorie del potenziale e dell'elasticità, 1884, in [2] 4, p. 136-179

[7] BELTRAMI, E : Sulla rappresentazione delle forze newtoniane per mezzo di forze elastiche, 1884 , in [2] 4, p. $95-103$

[8] BELTRAMI, E : Sull'interpretazione meccanica delle formole di Maxwell, 1884, in [2] 4, p. $190-223$

[9] BETTI, E : Teorica delle forze newtoniane e sue applicazioni all'elettrostatica e al magnetismo, Pisa, Nistri, 1879

[10] BOI, L, GIACARDI, L, TAZZIOLI, R (ed. by): La découverte de la géométrie non euclidienne sur la pseudosphère, Paris, Blanchard, 1998

[11] BotTAZZINI, U : Enrico Betti e la formazione della Scuola Matematica Pisana, Atti del Convegno La Storia delle Matematiche in Italia, Cagliari, 29-30 settembre -1 ottobre 1982, p. 229-275.

[12] BOTTAZZINI, U : Va' pensiero. Immagini della matematica nell'Italia dell'Ottocento, Bologna, Il Mulino, 1994

[13] BOTTAZZINI, U : Brioschi e gli Annali di Matematica, in [27], p. 71- 84

[14] BRIgaglia, A, MasotTo, G : Il Circolo Matematico di Palermo, Bari, Dedalo, 1982

[15] BRIOSCHI, F : La teorica dei determinanti e le loro principali applicazioni, Pavia, 1854

[16] CASORATI, F : Teorica delle funzioni di varibili complesse, Pavia, Fratelli Fusi, 1868

[17] CASTEllana, M, PALladino, F (ed. by): Giuseppe Battaglini. Raccolta di lettere (18541891) di un matematico al tempo del Risorgimento d'Italia, Bari, Levante, 1996

[18] CHELINI, D : Elementi di meccanica razionale con Appendice sui principii fondamentali delle matematiche, Bologna, G. Legnani, 1860

[19] COOKE, R : The Mathematics of Sonya Kovalevskaya, Springer, 1984

[20] CREMONA, L : Le figure reciproche della statica grafica, Milano, Hoepli, 1872

[21] DARRIGOL, O : Electrodynamics from Ampère to Einstein, Oxford, Oxford University Press, 2000

[22] DINI, U : Fondamenti per la teorica delle funzioni di variabili reali, Pisa, Nistri, 1878 
[23] ENEA, M R (ed. by) : Il Carteggio Beltrami-Chelini (1863-1873), Milano, Mimesis, 2009

[24] GATTO, R (ed. by) : Lettere di Luigi Cremona a Enrico Betti (1860-1890), in La corrispondenza di Luigi Cremona (1830-1903), vol. III (M. Menghini, ed.), Quaderno Pristem N. 9, Palermo, 1996, p. $7-90$

[25] GIACARDI, L, TAZZIOLI, R (ed. by) : "Pel lustro della Scienza italiana e per progresso dell'alto insegnamento". Le lettere di Beltrami a Betti, Tardy e Gherardi, Milano, Mimesis, to appear 2011

[26] GRAF,J H (ed. by), La correpondance entre Ludwig Schläfli et des Mathématiciens Italiens de son époque, Bollettino di Bibliografia e Storia delle Scienze Matematiche, 17, 1915, p. 81-86, p. 113-122

[27] LACAITA, C G, SILVESTRI, A (ed. by) : Francesco Brioschi e il suo tempo (1824-1897), Milano, Franco Angeli, 2000

[28] LORIA, G : Eugenio Beltrami e le sue opere matematiche, Bibliothca Mathmatica, s. 2, 3, 1901, p. $392-440$

[29] MAIOCCHI, R : Il Politecnico di Francesco Brioschi, in [?], p. 51-69

[30] MAXWELL, J C, A Treatise on Electricity and Magnetism, 2 vols., Oxford, Clarendon, 1873

[31] MICHELACCI, G (ed. by) : Le lettere di Charles Hermite ad Angelo Genocchi (1868-1887), Quaderni matematici, Università degli Studi di Trieste, 546, 2003.

[32] PALlADINO, F, TAZZIOLI, R : Le lettere di Eugenio Beltrami nella corrispondenza di Ernesto Cesro, Archive for History of Exact Sciences, 49, 1996, p. 321-353

[33] PEPE, L : Universitari italiani nel Risorgimento, Bologna, Clueb, 2002

[34] RIEMANN, B : Fondamenti di una teorica generale delle funzioni di una variabile complessa (transl. by E. Betti), Annali di matematica pura ed applicata, s. 1, 2, 1859, p. 288-304, p. $337-356$

[35] TAZZIOLI, R : Ether and Theory of Elasticity in Betrami's Work, Archive for History of Exact Sciences, 46, 1993, p.1-37

[36] TAZZIOLI, R : Rudolph Lipschitz's Work on Differential Geometry and Mechanics, in The History of Modern Mathematics (E. Knobloch and D.E. Rowe eds.), Academic Press, 1994, p. $113-138$

[37] TAZZIOLI, R, Beltrami e I matematici relativisti. La meccanica in spazi curvi nella seconda metà dell'Ottocento, Quaderni dell'Unione Matematica Italiana, 47, Bologna, Pitagora, 2000

[38] VOIGT, W : Elementare Mechanik als Einleitung in das Studium der theoretischen Physik, Leipzig, Veit, 1889. Italian Transl. Meccanica elementare, Rome, Loescher, 1894

[39] VOLTERRA, V : Le matematiche in Italia nella seconda metà del secolo XIX, in [40], 55-79

[40] VOLTERRA, V : Saggi scientifici, Bologna, Zanichelli, 1920 